\title{
DISCRETIZATION OF VECTOR BUNDLES AND ROUGH LAPLACIAN*
}

\author{
TATIANA MANTUANO ${ }^{\dagger}$
}

\begin{abstract}
Let $\mathcal{M}\left(m, \kappa, r_{0}\right)$ be the set of all compact connected $m$-dimensional manifolds $(M, g)$ such that $\operatorname{Ricci}(M, g) \geq-(m-1) \kappa g$ and $\operatorname{Inj}(M, g) \geq r_{0}>0$. Let $\mathcal{E}\left(n, k_{1}, k_{2}\right)$ be the set of all Riemannian vector bundles $(E, \nabla)$ of real rank $n$ with $\left|R^{E}\right| \leq k_{1}$ and $\left|d^{*} R^{E}\right| \leq k_{2}$. For any vector bundle $E \in \mathcal{E}\left(n, k_{1}, k_{2}\right)$ with harmonic curvature or with complex rank one, over any $M \in$ $\mathcal{M}\left(m, \kappa, r_{0}\right)$ and for any discretization $X$ of $M$ of mesh $0<\varepsilon \leq \frac{1}{20} r_{0}$, we construct a canonical twisted Laplacian $\Delta_{A}$ and a potential $V$ depending only on the local geometry of $E$ and $M$ such that we can compare uniformly the spectrum of the rough Laplacian $\bar{\Delta}$ associated to the connection of $E$ and the spectrum of $\Delta_{A}+V$. We show that there exist constants $c, c^{\prime}>0$ depending only on the parameters of $\mathcal{M}\left(m, \kappa, r_{0}\right)$ and $\mathcal{E}\left(n, k_{1}, k_{2}\right)$ such that $c^{\prime} \lambda_{k}(X, A, V) \leq \lambda_{k}(E) \leq c \lambda_{k}(X, A, V)$, where $\lambda_{k}(\cdot)$ denotes the $k^{t h}$ eigenvalue of the considered operators $(k \leq n|X|)$. For flat vector bundles, we show that the potential is zero, $\Delta_{A}$ turns out to be a discrete magnetic Laplacian and we relate $\lambda_{1}(E)$ to the holonomy of $E$.
\end{abstract}

Key words. Connection, rough Laplacian, discrete magnetic Laplacian, Harper operator, eigenvalues, discretization, holonomy

AMS subject classifications. 58J50, 53C20

1. Introduction. In [22], we have shown that for a family of compact connected manifolds $\mathcal{M}\left(m, \kappa, r_{0}\right)$ with injectivity radius and Ricci curvature bounded below (i.e. $(M, g) \in \mathcal{M}\left(m, \kappa, r_{0}\right)$ if $M$ is a compact connected $m$-dimensional Riemannian manifold with $\operatorname{Ricci}(M, g) \geq-(m-1) \kappa g$ and $\left.\operatorname{Inj}(M, g) \geq r_{0}\right)$, we can compare uniformly the spectrum of the Laplacian acting on functions with the spectrum of the combinatorial Laplacian acting on a graph with fixed mesh constructed on the manifolds. Indeed, we show that there exist positive constants $c, c^{\prime}$ depending on the parameters of the problem such that for any $M \in \mathcal{M}\left(m, \kappa, r_{0}\right)$ and any discretization $X$ of $M$ (with mesh $\varepsilon<\frac{1}{2} r_{0}$ ), the following holds

$$
c^{\prime} \lambda_{k}(X) \leq \lambda_{k}(M) \leq c \lambda_{k}(X)
$$

for $k<|X|$, where $\lambda_{k}(\cdot)$ stands for the $k^{\text {th }}$ eigenvalue of the considered Laplacian. This result generalizes in a natural way different works like [5], [6], [9] and [19] that were motivated either by the study of the relation between the fundamental group of a manifold and the spectrum of its finite coverings ([5], [6]) or by the relation between the spectrum of a manifold and its Cheeger isoperimetric constant ([9]) or by the existence of harmonic functions ([19]). More generally, the aim of the discretization is to have an understanding of the spectrum (a global invariant on the manifold) with a minimum of informations about the local geometry of the manifold.

Of course, the problem is interesting for differential operators other than the Laplacian and we may address the following question: does the same kind of comparison hold for other geometric differential operators such that the Laplacian acting on $p$-forms or the Dirac operator? Most of these operators may be expressed in terms of a connection Laplacian added with a curvature term. In this article, we investigate

\footnotetext{
${ }^{*}$ Received October 24, 2006; accepted for publication November 20, 2006.

†Université de Neuchâtel, Institut de Mathématiques, rue Emile-Argand 11, 2009 Neuchâtel, Switzerland (Tatiana.Mantuano@unine.ch). Supported by Swiss National Science Foundation, grant No. 20-109130.
} 
the case of such a connection (or rough) Laplacian $\bar{\Delta}$ associated to a connection $\nabla$ on a vector bundle. More precisely, the purpose is to establish a uniform comparison of spectra between rough Laplacians on vector bundles and twisted Laplacians on graphs that generalize combinatorial or discrete magnetic Laplacians. The Riemannian vector bundles we are interested in have curvature and exterior coderivative of curvature bounded i.e. we study Riemannian vector bundles $E$ with fiber of real rank $n$ such that $\left|R^{E}\right| \leq k_{1}$ and $\left|d^{*} R^{E}\right| \leq k_{2}$ (denote by $\mathcal{E}\left(n, k_{1}, k_{2}\right)$ the set of such vector bundles). The main result (Theorem 3.1) states that there exist positive constants $c, c^{\prime}$ (depending only on the given parameters) such that for any vector bundle $E \in \mathcal{E}\left(n, k_{1}, k_{2}\right)$ over any $M \in \mathcal{M}\left(m, \kappa, r_{0}\right)$ satisfying one of the following assumptions

I) the curvature of $E$ is harmonic i.e. $d^{*} R^{E}=0$,

II) $E$ is of complex (or quaternionic) rank one

and for any discretization $X$ of $E$, we can construct a canonical twisted Laplacian $\Delta_{A}$ and a potential $V$ depending only on the local geometry of $E$ such that

$$
c^{\prime} \lambda_{k}(X, A, V) \leq \lambda_{k}(E) \leq c \lambda_{k}(X, A, V)
$$

for any $k \leq n|X|$, where $\lambda_{k}(E)$ denotes the $k^{\text {th }}$ eigenvalue of the rough Laplacian $\bar{\Delta}$ and $\lambda_{k}(X, A, V)$ the $k^{\text {th }}$ eigenvalue of $\Delta_{A}+V$.

The case of flat vector bundles is especially enlightening. Indeed, if $E$ is flat, we show that the potential $V$ is zero and that $\Delta_{A}$ is a discrete magnetic Laplacian. This particular case shows how the construction of $\Delta_{A}$ is strongly related to the holonomy of $E$. This fact is emphasized by Theorem 4.1 which relates the holonomy (in the sense of [2]) to the first eigenvalue of $\Delta_{A}$ and therefore of $\bar{\Delta}$. In order to understand the problem of non-flat vector bundles, go back to the case of functions. Recall that for functions we needed to establish correspondances between functions on the manifold and functions on the graph. To that aim and in particular to associate smooth functions to functions on the graph, we had to extend locally such a function in a constant way and then smooth it (with a partition of unity). The question of extending locally is a central problem for the case of vector bundles. It turns out that extending by parallel transport is really efficient for flat vector bundles as it produces parallel sections. But, as soon as the curvature comes in, parallel transport is not convenient anymore and we need to construct a finer way to extend locally a section. In fact, the obstruction to extend in a parallel manner is double: the holonomy plays the role of a global obstruction to extend as parallel as possible and locally the curvature plays the same role. The twisted Laplacian will precisely render the holonomy of the vector bundle, while the potential will take into account the local non-flat geometry.

The paper is organized as follows. In Section 2, we introduce the notations, we define the general notion of twisted Laplacian on a graph and recall the main properties of the discretization of a manifold (that will coincide with the notion of discretization of vector bundles). Section 3 is devoted to the proof of the main result (Theorem 3.1). The main difficulty is to construct a suitable twisted Laplacian (see Section 3.1). From a geometric point of view, the problem is the dependence on the local geometry of the Laplacian and the potential to have enough informations to estimate the spectrum of the vector bundle. Technically, we need fine analysis on vector bundles like Sobolev inequalities for sections to achieve the construction. The particular case of flat vector bundles can be kept in mind as the ground example during the reading. In this case, the proofs can be done easier (we can avoid the 
technical tools described in Section 3.1). Nevertheless, this case already contains the essential information for $\Delta_{A}$ as it shows how the holonomy is related to $\Delta_{A}$ (see Section 4). For non-flat vector bundles, $\Delta_{A}$ does not suffice anymore to control the rough Laplacian, so that we have to introduce a potential $V$ which takes care of the curvature locally. The generalization of the flat case is then done for two different cases (see assumptions I) and II)), for rank one vector bundles and for vector bundles with harmonic curvature. These two cases are really of different nature. This appears all along Section 3 and this begins with the construction of $\Delta_{A}+V$ (in Section 3.2) which differs according to the assumptions I) or II). In Section 4, we establish the relationship between the holonomy and the first eigenvalue of the rough Laplacian for flat vector bundles. The part of Theorem 4.1 that bounds from below the first eigenvalue in terms of the holonomy can be generalized easily to vector bundles with harmonic curvature. But this will not be done here. This result is in fact due to Ballmann, Brüning and Carron in a more general setting (see [2]). Finally, we collect some more technical proofs in the appendix to make easier the reading, even if the results are not of minor importance for the paper.

\section{Settings.}

2.1. Rough Laplacian. In this section, we recall basic facts on the rough Laplacian (for a general reference see [3], [24] or [25] for instance). Let $(M, g)$ be a compact connected $m$-dimensional Riemannian manifold without boundary and with volume form denoted by $d V$. Moreover, let $(E, \nabla)$ be a Riemannian vector bundle with $n$ dimensional fiber over $M$ i.e. $E$ is a vector bundle over $M$ endowed with a smooth metric $\langle\cdot, \cdot \cdot\rangle$ and a compatible connection $\nabla$. On the set $\Gamma(E)$ of smooth sections of $E$, denote by $(\cdot, \cdot)$ the $L^{2}$-inner product endowed by $\langle\cdot, \cdot\rangle$ and $g$. Recall that the connection extends to $p$-tensors on $M$ with values in $E$ and that we define $\nabla^{*}$ to be the adjoint of $\nabla$ with respect to the $L^{2}$-inner product. The rough Laplacian (or connection Laplacian) acting on $\Gamma(E)$ is then defined by $\bar{\Delta}=\nabla^{*} \nabla$. The spectrum of $\bar{\Delta}$ is discrete and non-negative and will be denoted

$$
\operatorname{Spec}(E)=\left\{\lambda_{1}(E) \leq \lambda_{2}(E) \leq \ldots \leq \lambda_{k}(E) \leq \ldots\right\}
$$

The Rayleigh quotient of a non-zero section $s$ is defined by $R(s)=\frac{\|\nabla s\|^{2}}{\|s\|^{2}}$, where $\|\cdot\|$ denotes the $L^{2}$-norm associated to the $L^{2}$-inner product defined above. Later we will need the following variational characterizations of $\operatorname{Spec}(E)$ known as min-max and max-min theorems. For any $k \geq 1$,

$$
\begin{aligned}
\lambda_{k}(E) & =\min _{\Omega^{k}} \max \left\{R(s): s \in \Omega^{k} \backslash\{0\}\right\} \\
& =\max _{\Omega^{k-1}} \min \left\{R(s): s \in \Omega^{k-1} \backslash\{0\}, s \perp \Omega^{k-1} \text { w.r.t }(\cdot, \cdot)\right\}
\end{aligned}
$$

where $\Omega^{k}$ (resp. $\Omega^{k-1}$ ) ranges over all $k$-dimensional (resp. $(k-1)$-dim.) subspaces of $\Gamma(E)$.

2.2. Twisted Laplacian. Let $\Gamma=(X, E(X))$ be a finite connected graph endowed with the path metric. For $p \in X$ denote by $N(p)$ the set of vertices at distance 1 from $p$ and by $m(p)$ the number of such vertices. In order to generalize the combinatorial Laplacian (see [21] for a definition) and the discrete magnetic Laplacian (see [23] for a definition), let us consider the set of functions on $X$ with values in $\mathbb{R}^{n}$ i.e. $\mathcal{F}(X)=\left\{f: X \rightarrow \mathbb{R}^{n}\right\}$, provided with the inner product $(f, g)=\sum_{p \in X} f(p) \cdot g(p)$, where $\cdot$ denotes the Euclidean inner product of $\mathbb{R}^{n}$. 
Definition 2.1. For any $p \in X$ and $q \in N(p)$ assume that $A(p, q): \mathbb{R}^{n} \rightarrow \mathbb{R}^{n}$ is a given linear transformation. The twisted Laplacian associated to $A$ is the operator $\Delta_{A}: \mathcal{F}(X) \rightarrow \mathcal{F}(X)$ defined by

$$
\Delta_{A} f(p)=\frac{1}{2} \sum_{q \in N(p)}\left(\mathbb{I}+A^{t}(p, q) A(p, q)\right) f(p)-\left(A(q, p)+A^{t}(p, q)\right) f(q) .
$$

REMARK 2.2. If for any $p, q$, the operator $A(p, q)$ is the identity, then $\Delta_{A}$ is the combinatorial Laplacian.

REMARK 2.3. If $A(p, q)$ belongs to $O(n)$ and $A^{t}(p, q)=A(q, p)$, then $\Delta_{A} f(p)=m(p) f(p)-\sum_{q \in N(p)} A(q, p) f(q)$. In this case the twisted Laplacian is usually called discrete magnetic Laplacian or Laplacian associated to the Harper operator $A$.

Let us introduce the space of functions $\mathcal{F}(X \times X)=\left\{F: X \times X \rightarrow \mathbb{R}^{n}\right\}$ and provide it with the inner product given by $(F, G)=\frac{1}{2} \sum_{p \in X} \sum_{q \in X} F(p, q) \cdot G(p, q)$.

Lemma 2.4. Let $A(p, q)$ be as in Definition 2.1 and $\Delta_{A}$ the twisted Laplacian associated to $A$. Let $D_{A}: \mathcal{F}(X) \rightarrow \mathcal{F}(X \times X)$ be defined by

$$
D_{A} f(p, q)= \begin{cases}f(q)-A(p, q) f(p) & \text { if } p \in N(q) \\ 0 & \text { otherwise }\end{cases}
$$

Then, for any $f, g \in \mathcal{F}(X)$, we have $\left(\Delta_{A} f, g\right)=\left(D_{A} f, D_{A} g\right)$.

Proof. Let $f, g \in \mathcal{F}(X)$. Then, we have

$$
\begin{aligned}
\left(\Delta_{A} f, g\right)= & \frac{1}{2} \sum_{p \in X} \sum_{q \in N(p)}(f(p)-A(q, p) f(q)) \cdot g(p) \\
& -\frac{1}{2} \sum_{p \in X} \sum_{q \in N(p)}(f(q)-A(p, q) f(p)) \cdot A(p, q) g(p) \\
= & \frac{1}{2} \sum_{p \in X} \sum_{q \in X} D_{A} f(q, p) \cdot g(p)+\frac{1}{2} \sum_{p \in X} \sum_{q \in X} D_{A} f(p, q) \cdot D_{A} g(p, q) \\
& -\frac{1}{2} \sum_{p \in X} \sum_{q \in X} D_{A} f(p, q) \cdot g(q)=\left(D_{A} f, D_{A} g\right) .
\end{aligned}
$$

A direct consequence of this lemma is that $\Delta_{A}$ is symmetric and non-negative, so it admits a non-negative spectrum. If $V: \mathcal{F}(X) \rightarrow \mathcal{F}(X)$ is a non-negative potential, then the spectrum of $\Delta_{A}+V$ is characterized by min-max theorem as follows

$$
\forall 1 \leq k \leq n|X|, \quad \lambda_{k}(X, A, V)=\min _{W^{k}} \max \left\{R(f): f \in W^{k} \backslash\{0\}\right\}
$$

where $W^{k}$ ranges over all $k$-dimensional vector subspaces of $\mathcal{F}(X)$ and $R(f)$ is the Rayleigh quotient of $f$ defined by $R(f)=\frac{\left\|D_{A} f\right\|^{2}+(V f, f)}{\|f\|^{2}}$. 
2.3. Discretization of vector bundles. In this section, we define the notion of discretization of a vector bundle.

Definition 2.5. Let $(E, \nabla)$ be a Riemannian vector bundle over $(M, g)$ a compact connected Riemannian manifold with $\partial M=\emptyset$. An $\varepsilon$-discretization of $E$ is a discretization of $M$ of mesh $\varepsilon>0$.

The discretization of a manifold (of mesh $\varepsilon$ ) is defined as in [10] (Section V.3.2). Let us recall the definition and the properties of such a discretization. Let $(M, g)$ be a compact connected $m$-dimensional Riemannian manifold. A discretization of $M$, of mesh $\varepsilon>0$, is a maximal $\varepsilon$-separated subset $X$ of $M$ provided with a graph structure given by the sets $N(p)=\{q \in X \mid 0<d(p, q)<3 \varepsilon\}$, for any $p \in X$. In other words, $X$ is such that for any distinct $p, q \in X, d(p, q) \geq \varepsilon$ and $\bigcup_{p \in X} B(p, \varepsilon)=M$. Moreover, $p q$ is an edge if and only if $0<d(p, q)<3 \varepsilon$. Denote by $m(p)$ the number of elements of $N(p)$.

REMARK 2.6. Let us remark that if $B(p, \rho)$ is a ball in $M$ with radius $\rho<$ $\frac{1}{2} \operatorname{Inj}(M)$, then the volume $V(p, \rho)$ of the ball $B(p, \rho)$ is bounded below by a constant depending only on $\rho$ and $m$ (this is Croke's Inequality, see for instance in [10] p.136). Moreover, if $M$ has Ricci curvature bounded below by $-(m-1) \kappa$ then the volume of a ball of radius $R$ is bounded above by a constant depending only on $m, \kappa$ and $R$ (this follows from Bishop's comparison theorem, see for instance [10], p.126). These bounds will be used frequently in the sequel.

Choose $\varepsilon$ smaller than $\frac{1}{2} \operatorname{Inj}(M)$. Denote by $\kappa \geq 0$ a constant such that $\operatorname{Ricci}(M, g) \geq-(m-1) \kappa g$. Then, using Remark 2.6 we can show that $m(p)$ is bounded above by a constant $\nu_{X}$ depending only on $m, \kappa$ and $\varepsilon$ and that $\frac{1}{V_{-\kappa}(\varepsilon)} \operatorname{Vol}(M) \leq|X| \leq \frac{2^{m}}{\varepsilon^{m} c(m)} \operatorname{Vol}(M)$, where $V_{-\kappa}(\varepsilon)$ denotes the volume of the ball of radius $\varepsilon$ in the simply connected space of constant sectional curvature $-\kappa$ and of dimension $m$.

3. Spectra comparison for rough Laplacian and twisted Laplacian. In this section, we will establish the comparison between the spectra of the rough Laplacian and a twisted Laplacian. Let us state the main result.

Theorem 3.1. Let $m, n$ be positive integers, $\kappa, k_{1}, k_{2} \geq 0$ and $r_{0} \geq 20 \varepsilon>0$. There exist positive constants $c, c^{\prime}$ depending only on $m, n, \kappa, k_{1}, k_{2}$ and $\varepsilon$ such that for any $M \in \mathcal{M}\left(m, \kappa, r_{0}\right)$, any vector bundle $E \in \mathcal{E}\left(n, k_{1}, k_{2}\right)$ over $M$ satisfying one of the following condition

I) the curvature of $E$ is harmonic i.e. $d^{*} R^{E}=0$,

II) $E$ is of complex (or quaternionic) rank one

and for any $\varepsilon$-discretization $X$ of $E$, we can construct a canonical twisted Laplacian $\Delta_{A}$ and a potential $V$ depending only on the local geometry of $E$ such that, for $1 \leq$ $k \leq n|X|$

$$
c^{\prime} \lambda_{k}(X, A, V) \leq \lambda_{k}(E) \leq c \lambda_{k}(X, A, V) .
$$

In particular, if the vector bundle is flat, the potential is zero and $\Delta_{A}$ is a discrete magnetic Laplacian.

Roughly speaking, the basic idea of the proof is the same as to prove the theorem of comparison of spectra between the Laplacian acting on functions and the combinatorial Laplacian ([22], Theorem 3.7). But a first fundamental difference between the functions and the vector bundles cases is the construction of the twisted 
Laplacian. Indeed, in [22] the combinatorial Laplacian appearing in Theorem 3.7 is canonically associated to the graph that discretizes the manifold. For vector bundles, such a canonical Laplacian on graphs does not obviously exist. Hence, a first step of the proof consists in constructing a suitable twisted Laplacian $\Delta_{A}$ and a potential $V$ (Section 3.2) that will depend only on the local geometry. The construction of $\Delta_{A}+V$ differs according to the assumptions I) and II). We will work with balls centered on $X$ and for both cases the construction of $\Delta_{A}$ relies essentially on changes of bases from a ball to a neighboring ball, but for vector bundles satisfying II) the definition of $\Delta_{A}$ is slightly harder. A more significant difference is the construction of the potential $V$. For rank one vector bundles, $V$ involves only the first eigenvalue of balls (with Neumann boundary condition), while in the other case, we will distinguish "small" eigenvalues of balls from "large" eigenvalues. In rank one vector bundles the $n$ first eigenvalues (of such a ball) are the same and correspond to the minimum of the energy, so that it will make easier the estimating of $V$.

After defining the twisted Laplacian and the potential, we follow the same way of proof as for the case of functions, but the underlying analysis is much more difficult. For instance, we need to establish some Sobolev inequalities for sections that requires fine tools of analysis as Moser's iteration and Sobolev inequalities for functions (Lemma A.1 in Appendix). The definition of the smoothing operator $\mathcal{S}$ and the discretizing operator $\mathcal{D}$ generalizes in some sense the similar operators defined by Chavel in [10] (Sections VI.5.1 and VI.5.2). Similarly, we establish norms estimations for these operators $\mathcal{S}$ and $\mathcal{D}$ (Propositions 3.18 and 3.21) in order to compare Rayleigh quotients of sections with Rayleigh quotients of functions on the discretization. Then, min-max theorem leads to the result for "small" eigenvalues. It suffices moreover to have upper bounds on the respective spectra (Lemma 3.23) to compare "large" eigenvalues and conclude the proof of Theorem 3.1 (Section 3.6).

3.1. Local extension. In this section we define a way to extend a section as parallel as possible. In the case of flat vector bundles parallel transport is the suitable tool, because of the lemma below. Let $\tau_{x, p}$ denotes the parallel transport from $E_{p}$ to $E_{x}$ along the minimizing geodesic joining $p$ to $x$ (for $d(p, x)<\frac{1}{2} \operatorname{Inj}(M)$ ).

LEMma 3.2. Let $(E, \nabla)$ be a flat Riemannian vector bundle over a Riemannian manifold $(M, g)$. Let $p \in M$ and $B(p, r)$ the ball centered at $p$ of radius $r<\frac{1}{2} \operatorname{Inj}(M)$. Then for any $v \in E_{p}$, the section $\sigma$ over $B(p, r)$ defined by $\sigma(x)=\tau_{x, p} v$ is parallel.

Proof. See [12] Section 2.2.1.

In the non-flat case, extending by parallel transport is not strong enough for our purpose, because we need to control the covariant derivative of such extended sections. More precisely, we want to extend in an energy minimizing way. This means that we have to take into account local small eigenvalues. Hence, we introduce eigensections of the Neumann problem on balls which give an obstruction to extension in a parallel way. Such eigensections on balls associated to small eigenvalues are almost parallel (Lemma 3.3) and will provide a good way to extend sections. Nevertheless, it may happen that there are no (or only a few) small eigensections on a ball. In this case, parallel transport will be good enough to extend as we will see.

Lemma 3.3. Let $(E, \nabla) \in \mathcal{E}\left(n, k_{1}, k_{2}\right)$ over $(M, g) \in \mathcal{M}\left(m, \kappa, r_{0}\right)$. For $0<r<$ $\frac{1}{2} r_{0}$ and $p \in M$, let $\sigma: B(p, r) \rightarrow E$ be a section such that $\bar{\Delta} \sigma=\lambda \sigma$ for a constant $\lambda \geq 0$. Let $0<\theta<1$. Then there exist $0<c(m) \leq s \leq 1$ and $c, c^{\prime}>0$ depending on 
an upper bound for $\lambda$ and on $m, n, \kappa, r, k_{1}, k_{2}$ and $\theta$ such that

$$
\begin{aligned}
\|\sigma\|_{\infty, \theta r} & \leq c\|\sigma\|_{2, r}, \\
\|\nabla \sigma\|_{\infty, \theta r} & \leq c^{\prime}\|\nabla \sigma\|_{2, r}^{s}
\end{aligned}
$$

where $\|\cdot\|_{q, \rho}$ denotes the $L^{q}$-norm on the ball centered at $p$ of radius $\rho\left(c^{\prime}\right.$ depends on $c\|\sigma\|_{2, r}$ too $)$.

Moreover, there exists $c^{\prime \prime}>0$ depending on $c, c^{\prime}$ and $r$ such that

$$
\left|\sigma(x)-\tau_{x, p} \sigma(p)\right| \leq c^{\prime \prime}\|\nabla \sigma\|_{2, r}^{s}
$$

for all $x \in B(p, \theta r)$. If $k_{2}=0$ i.e. if $E$ is of harmonic curvature, then $s=1$ in the previous inequalities.

Proof. The idea is to use a Moser iteration to prove the statement. The more technical part of the argument is carried out in the appendix (see Lemma A.1). In order to use Lemma A.1, let $\delta>0$ and $u_{\delta}: B(p, r) \rightarrow \mathbb{R}$ defined by $u_{\delta}=\sqrt{|\sigma|^{2}+\delta}$. Then in one hand $\Delta\left(u_{\delta}^{2}\right)=2 u_{\delta} \Delta u_{\delta}-2\left|d u_{\delta}\right|^{2}$ and in the other hand $\Delta\left(u_{\delta}^{2}\right)=2\langle\sigma, \bar{\Delta} \sigma\rangle-$ $2|\nabla \sigma|^{2}$ which implies that

$$
u_{\delta} \Delta u_{\delta} \leq\langle\sigma, \bar{\Delta} \sigma\rangle=\lambda|\sigma|^{2} \leq \lambda u_{\delta}^{2} .
$$

We can then apply Lemma A.1 to $u_{\delta}$ and we get that $\left\|u_{\delta}\right\|_{\infty, \theta r} \leq c\left\|u_{\delta}\right\|_{2, r}$. Then let $\delta \rightarrow 0$ to obtain the first claim.

For the second inequality, let $\delta>0$ and $v_{\delta}: B(p, r) \rightarrow \mathbb{R}$ defined by $v_{\delta}(x)=$ $\sqrt{|\nabla \sigma(x)|^{2}+\delta}$. Then

$$
\Delta\left(v_{\delta}^{2}\right)=2 v_{\delta} \Delta v_{\delta}-2\left|d v_{\delta}\right|^{2}=2\langle\nabla \sigma, \bar{\Delta}(\nabla \sigma)\rangle-2|\nabla \nabla \sigma|^{2} .
$$

But we have that $|\nabla \nabla \sigma|^{2}-\left|d v_{\delta}\right|^{2} \geq 0$ and therefore

$$
v_{\delta} \Delta v_{\delta} \leq\langle\nabla \sigma, \bar{\Delta}(\nabla \sigma)\rangle=\langle\nabla \sigma, \bar{\Delta}(\nabla \sigma)-\nabla(\bar{\Delta} \sigma)\rangle+\lambda|\nabla \sigma|^{2} .
$$

By a commuting argument (see [1], Lemma 2.3) we have for a local orthonormal frame $\left\{X_{i}\right\}_{i=1, \ldots, m}$ of $M$

$$
\begin{aligned}
& \langle\nabla \sigma, \bar{\Delta}(\nabla \sigma)-\nabla(\bar{\Delta} \sigma)\rangle= \\
& \lambda|\nabla \sigma|^{2}-\left\langle\nabla_{R i c(\cdot)} \sigma, \nabla \sigma\right\rangle-2 \sum_{i=1}^{m}\left\langle R^{E}\left(X_{i}, \cdot\right) \nabla_{X_{i}} \sigma, \nabla \sigma\right\rangle+\left\langle\left(d^{*} R^{E}\right) \sigma, \nabla \sigma\right\rangle
\end{aligned}
$$

and as $\operatorname{Ricci}(M, g) \geq-(m-1) \kappa g,\left|R^{E}\right| \leq k_{1}$ and $\left|d^{*} R^{E}\right| \leq k_{2}$ we then get

$$
\langle\nabla \sigma, \bar{\Delta}(\nabla \sigma)-\nabla(\bar{\Delta} \sigma)\rangle \leq\left(\lambda+(m-1) \kappa+2 n^{2} k_{1}\right)|\nabla \sigma|^{2}+n^{2} k_{2}|\sigma||\nabla \sigma| .
$$

By the first part of the proof, we obtain that on $B(p, \theta r)$

$$
\langle\nabla \sigma, \bar{\Delta}(\nabla \sigma)-\nabla(\bar{\Delta} \sigma)\rangle \leq\left(\lambda+(m-1) \kappa+2 n^{2} k_{1}\right)|\nabla \sigma|^{2}+n^{2} k_{2} c\|\sigma\|_{2, r}|\nabla \sigma|
$$

and this implies (on $B(p, \theta r)$ )

$$
\Delta v_{\delta} \leq\left(\lambda+(m-1) \kappa+2 n^{2} k_{1}\right) v_{\delta}+n^{2} k_{2} c\|\sigma\|_{2, r} .
$$


If $\theta^{\prime}<\theta$ we can apply Lemma A.1 to $v_{\delta}$ and let $\delta \rightarrow 0$ to obtain

$$
\|\nabla \sigma\|_{\infty, \theta^{\prime} r} \leq c^{\prime}\|\nabla \sigma\|_{2, \theta r}^{s} \leq c^{\prime}\|\nabla \sigma\|_{2, r}^{s} .
$$

Note that if $k_{2}=0$, then $s=1$ and $c^{\prime}$ does not depend on $c\|\sigma\|_{2, r}$. The two first inequalities in the statement are then true for any $\theta^{\prime}$ such that $0<\theta^{\prime}<\theta<1$. So rename $\theta^{\prime}$ by $\theta$ to obtain the statement.

Finally, recall that if $\gamma$ is the minimizing geodesic joining $p$ to $x \in B(p, \theta r)$ of length $l(<\theta r)$, then $\left|\sigma(x)-\tau_{x, p} \sigma(p)\right| \leq \int_{0}^{l}\left|\nabla_{\dot{\gamma}(t)} \sigma(\gamma(t))\right| d t \leq l\|\nabla \sigma\|_{\infty, \theta r}$. Using (3.1) leads to the result. $\square$

From now on, let $E \in \mathcal{E}\left(n, k_{1}, k_{2}\right)$ over $M \in \mathcal{M}\left(m, \kappa, r_{0}\right)$ and fix $\varepsilon \leq \frac{1}{20} r_{0}$. Let $X$ be an $\varepsilon$-discretization of $E$. Let $\sigma_{k}^{p}: B(p, 10 \varepsilon) \rightarrow E$ be the eigensection associated to the $k^{\text {th }}$ eigenvalue $\lambda_{k}(p)$ of $\bar{\Delta}$ on $B(p, 10 \varepsilon)$ with Neumann boundary condition such that $\int_{B(p, 10 \varepsilon)}\left\langle\sigma_{k}^{p}, \sigma_{l}^{p}\right\rangle d V=\delta_{k l} V(p, 10 \varepsilon)$.

REMARK 3.4. If $E$ is flat $\lambda_{1}(p)=\ldots=\lambda_{n}(p)=0$ and the $\sigma_{k}^{p}$ 's give a local orthonormal frame over $B(p, 10 \varepsilon)$.

REMARK 3.5. If $n=2$ (resp. $n=4$ ) and $E$ is of complex (resp. quaternionic) rank one, then $\lambda_{1}(p)=\ldots=\lambda_{n}(p)$. Indeed, the section $i \sigma_{1}^{p}$ (resp. $i \sigma_{1}^{p}, j \sigma_{1}^{p}, k \sigma_{1}^{p}$ where $i, j, k$ are the quaternions with $i^{2}=j^{2}=k^{2}=$ -1) satisfies $\nabla\left(i \sigma_{1}^{p}\right)=i \nabla \sigma_{1}^{p}$ which implies that $i \sigma_{1}^{p}$ is a $\lambda_{1}(p)$-eigensection orthogonal to $\sigma_{1}^{p}$. Hence, we can choose $\sigma_{k}^{p}$ such that for any $x$ in $B(p, 10 \varepsilon)$, $\left\langle\sigma_{k}^{p}(x), \sigma_{l}^{p}(x)\right\rangle=0$ for any $1 \leq k \leq n, 1 \leq l \leq n, k \neq l$.

Lemma 3.6. Let $0 \leq \alpha<\frac{1}{n+1}$. There exists $\delta>0$ depending only on $\alpha, m$, $n, k_{1}, k_{2}, \kappa, \varepsilon$ such that if $\lambda_{k}(p) \leq \delta$ then $\forall 1 \leq i, j \leq k$ and $\forall x \in B(p, 8 \varepsilon)$ $\left|\left\langle\sigma_{i}^{p}(x), \sigma_{j}^{p}(x)\right\rangle-\delta_{i j}\right| \leq \alpha$. In particular, if $\lambda_{k}(p) \leq \delta$, then $\left\{\sigma_{1}^{p}(x), \ldots, \sigma_{k}^{p}(x)\right\}$ spans a $k$-dimensional vector subspace of $E_{x}$, for any $x \in B(p, 8 \varepsilon)$.

To prove this lemma, let us recall a basic fact of linear algebra (the proof of the fact is left to the reader). Let $V$ be an $n$-dimensional vector space provided with an inner product $\langle\cdot, \cdot\rangle$. If $\left\{v_{1}, \ldots, v_{n}\right\} \subseteq V$ is such that $\left|\left\langle v_{i}, v_{j}\right\rangle-\delta_{i j}\right| \leq \alpha<$ $\frac{1}{n+1}$, then $\left\{v_{1}, \ldots, v_{n}\right\}$ is a basis of $V$. Moreover for any $v=\sum_{i=1}^{n} a_{i} v_{i}$, we have $(1-\alpha(n+1)) \sum_{i=1}^{n} a_{i}^{2} \leq\|v\|^{2} \leq(1+\alpha(n+1)) \sum_{i=1}^{n} a_{i}^{2}$. Such a basis will be referred as an almost orthonormal basis.

Proof of Lemma 3.6. Let $f_{i j}(x)=\left\langle\sigma_{i}^{p}(x), \sigma_{j}^{p}(x)\right\rangle$ and denote by $m_{i j}$ its mean over $B(p, 10 \varepsilon)$, then

$$
m_{i j}=\frac{1}{V(p, 10 \varepsilon)} \int_{B(p, 10 \varepsilon)} f_{i j} d V=\delta_{i j} .
$$

A result of Kanai ensuring the existence of $c_{K}>0$ depending only on $\varepsilon$ and $\kappa$ (see [10], Lemma VI.5.5) and the assumption $\lambda_{k}(p) \leq \delta$ imply

$$
0 \leq \int_{B(p, 10 \varepsilon)}\left|f_{i j}-\delta_{i j}\right| d V \leq c_{K} \int_{B(p, 10 \varepsilon)}\left|d f_{i j}\right| d V \leq c_{K} V(p, 10 \varepsilon) \sqrt{\delta}
$$

Moreover,

$$
\begin{aligned}
\inf _{x \in B\left(p, \frac{\varepsilon}{2}\right)}\left\{\left|f_{i j}(x)-\delta_{i j}\right|\right\} V\left(p, \frac{\varepsilon}{2}\right) & \leq \int_{B\left(p, \frac{\varepsilon}{2}\right)}\left|f_{i j}(x)-\delta_{i j}\right| d V(x) \\
& \leq c_{K} V(p, 10 \varepsilon) \sqrt{\delta} .
\end{aligned}
$$


The last inequality follows from (3.2). Hence (3.3) implies that there exists $p^{\prime} \in$ $M, d\left(p, p^{\prime}\right) \leq \frac{\varepsilon}{2}$, such that

$$
\left|\left\langle\sigma_{i}^{p}\left(p^{\prime}\right), \sigma_{j}^{p}\left(p^{\prime}\right)\right\rangle-\delta_{i j}\right| \leq 2 c_{K} \frac{V(p, 10 \varepsilon)}{V\left(p, \frac{\varepsilon}{2}\right)} \sqrt{\delta} \leq c \sqrt{\delta} .
$$

We conclude then as follows

$$
\begin{aligned}
\left|\left\langle\sigma_{i}^{p}(x), \sigma_{j}^{p}(x)\right\rangle-\delta_{i j}\right| & \leq \\
\mid\left\langle\sigma_{i}^{p}(x), \sigma_{j}^{p}(x)\right\rangle- & \left\langle\tau_{x, p^{\prime}} \sigma_{i}^{p}\left(p^{\prime}\right), \tau_{x, p^{\prime}} \sigma_{j}^{p}\left(p^{\prime}\right)\right\rangle|+|\left\langle\sigma_{i}^{p}\left(p^{\prime}\right), \sigma_{j}^{p}\left(p^{\prime}\right)\right\rangle-\delta_{i j} \mid \\
& \leq\left|\left\langle\sigma_{i}^{p}(x), \sigma_{j}^{p}(x)\right\rangle-\left\langle\tau_{x, p^{\prime}} \sigma_{i}^{p}\left(p^{\prime}\right), \tau_{x, p^{\prime}} \sigma_{j}^{p}\left(p^{\prime}\right)\right\rangle\right|+c \sqrt{\delta} .
\end{aligned}
$$

For any $x \in B(p, 8 \varepsilon)$ the minimizing geodesic $\overline{x p^{\prime}}$ stays in $B(p, 9 \varepsilon)$, so we can write

$$
\begin{aligned}
\mid\left\langle\sigma_{i}^{p}(x), \sigma_{j}^{p}(x)\right\rangle & -\left\langle\sigma_{i}^{p}\left(p^{\prime}\right), \sigma_{j}^{p}\left(p^{\prime}\right)\right\rangle \mid \leq 9 \varepsilon\left\|d\left\langle\sigma_{i}^{p}, \sigma_{j}^{p}\right\rangle\right\|_{\infty, 9 \varepsilon} \\
\leq 9 \varepsilon\left(\left\|\nabla \sigma_{i}^{p}\right\|_{\infty, 9 \varepsilon}\left\|\sigma_{j}^{p}\right\|_{\infty, 9 \varepsilon}+\left\|\sigma_{i}^{p}\right\|_{\infty, 9 \varepsilon}\left\|\nabla \sigma_{j}^{p}\right\|_{\infty, 9 \varepsilon}\right) & \leq 9 \varepsilon c^{\prime}\left(\left\|\nabla \sigma_{i}^{p}\right\|_{2,10 \varepsilon}^{s}\left\|\sigma_{j}^{p}\right\|_{2,10 \varepsilon}+\left\|\sigma_{i}^{p}\right\|_{2,10 \varepsilon}\left\|\nabla \sigma_{j}^{p}\right\|_{2,10 \varepsilon}^{s}\right)
\end{aligned}
$$

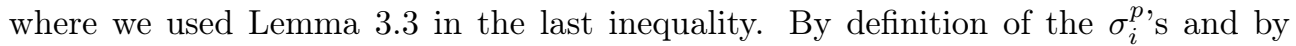
assumption on $\lambda_{i}(p)$ we get

$$
\left|\left\langle\sigma_{i}^{p}(x), \sigma_{j}^{p}(x)\right\rangle-\left\langle\sigma_{i}^{p}\left(p^{\prime}\right), \sigma_{j}^{p}\left(p^{\prime}\right)\right\rangle\right| \leq c^{\prime \prime} \sqrt{\delta^{s}} .
$$

Finally, (3.4) and (3.5) imply that for a sufficiently small $\delta$ we have

$$
\left|\left\langle\sigma_{i}^{p}(x), \sigma_{j}^{p}(x)\right\rangle-\delta_{i j}\right| \leq\left(c \sqrt{\delta}+c^{\prime \prime} \sqrt{\delta^{s}}\right) \leq \alpha<\frac{1}{n+1}
$$

and this ends the proof.

Definition 3.7. Fix once and for all $0<\alpha<\frac{1}{n+1}$. Let $\delta$ be given by Lemma 3.6. For $p \in X$, define then $\boldsymbol{\mu}(\boldsymbol{p})$ as the largest integer such that $\lambda_{\mu(p)}(p) \leq \delta$.

REMARK 3.8. If the vector bundle is flat, $\mu(p)=n$, for any $p \in X$.

For $p \in X$, we want to extend a section in a neighborhood of $p$ as parallel as possible and taking care of local small eigenvalues as said before. So let us define the local extension that associates to a vector in $E_{p}$ a local section over $B(p, 10 \varepsilon)$. Consider $E_{\mu(p)}$ the $\mu(p)$-dimensional vector subspace of $E_{p}$ spanned by $\left\{\sigma_{1}^{p}(p), \ldots, \sigma_{\mu(p)}^{p}(p)\right\}$. Let $E_{\mu(p)}^{\perp}$ be the orthogonal complement of $E_{\mu(p)}$ in $E_{p}$ and choose $\left\{e_{\mu(p)+1}^{p}, \ldots, e_{n}^{p}\right\}$ an orthonormal basis of $E_{\mu(p)}^{\perp}$. By construction, $\left\{e_{1}^{p}=\sigma_{1}^{p}(p), \ldots, e_{\mu(p)}^{p}=\sigma_{\mu(p)}^{p}(p), e_{\mu(p)+1}^{p}, \ldots, e_{n}^{p}\right\}$ is an almost orthonormal basis of $E_{p}$. We extend this basis on $B(p, 10 \varepsilon)$ by

$$
e_{i}^{p}(x):= \begin{cases}\sigma_{i}^{p}(x) & \text { if } i \leq \mu(p), \\ \tau_{x, p} e_{i}^{p} & \text { otherwise }\end{cases}
$$

and we define the local extension of $v=\sum_{i=1}^{n} v_{i} e_{i}^{p}$ by $\sum_{i=1}^{n} v_{i} e_{i}^{p}(x)$.

REMARK 3.9. If $E$ is flat, the local extension corresponds to the extension by parallel transport along radial geodesics. In this case, it suffices to choose any orthonormal basis $\left\{e_{1}^{p}, \ldots, e_{n}^{p}\right\}$ of $E_{p}$ and extend it radially to obtain $\left\{e_{1}^{p}(x), \ldots, e_{n}^{p}(x)\right\}$. 
Lemma 3.10. For any $x \in B(p, 8 \varepsilon),\left\{e_{1}^{p}(x), \ldots, e_{n}^{p}(x)\right\}$ is an almost orthonormal basis of $E_{x}$.

Proof. If $\mu(p)=0$ the claim is clearly true. If $\mu(p)=n$ the claim follows from Lemma 3.6. Hence suppose $1 \leq \mu(p) \leq n-1$. By Lemma 3.6 $\left\langle e_{1}^{p}(x), \ldots, e_{\mu(p)}^{p}(x)\right\rangle$ is $\mu(p)$-dimensional and as parallel translation preserves the inner product $\left\langle e_{\mu(p)+1}^{p}(x), \ldots, e_{n}^{p}(x)\right\rangle$ is $(n-\mu(p))$-dimensional. So we have to show that there exists $c>0$ such that

$$
\left|\left\langle e_{i}^{p}(x), e_{j}^{p}(x)\right\rangle\right| \leq c<\frac{1}{n+1}, \quad \forall 1 \leq i \leq \mu(p)<j \leq n .
$$

Let us prove this estimate. As $e_{j}^{p}(p)$ and $\sigma_{i}^{p}(p)$ are orthogonal, we have

$$
\begin{aligned}
\left|\left\langle e_{j}^{p}(x), e_{i}^{p}(x)\right\rangle\right| & =\left\langle e_{j}^{p}(p), \tau_{p, x} \sigma_{i}^{p}(x)-\sigma_{i}^{p}(p)\right\rangle \\
& \leq\left|e_{j}^{p}(p)\right| \cdot\left|\sigma_{i}^{p}(x)-\tau_{x, p} \sigma_{i}^{p}(p)\right|=\left|\sigma_{i}^{p}(x)-\tau_{x, p} \sigma_{i}^{p}(p)\right| .
\end{aligned}
$$

By Lemma $3.3\left|\sigma_{i}^{p}(x)-\tau_{x, p} \sigma_{i}^{p}(p)\right| \leq c^{\prime} \sqrt{\delta^{s}}$. Hence $\left|\left\langle e_{j}^{p}(x), e_{i}^{p}(x)\right\rangle\right| \leq c^{\prime} \sqrt{\delta^{s}}$. Then, readjust $\delta$ if necessary to obtain $\left|\left\langle e_{j}^{p}(x), e_{i}^{p}(x)\right\rangle\right| \leq c<\frac{1}{n+1}$.

REMARK 3.11. For the sequel, let $\delta^{\prime}$ denote a constant, $0<\delta^{\prime}<1$, such that $\left(1-\delta^{\prime}\right) \sum_{i=1}^{n} v_{i}^{2} \leq\left|\sum_{i=1}^{n} v_{i} e_{i}^{p}(x)\right|^{2} \leq\left(1+\delta^{\prime}\right) \sum_{i=1}^{n} v_{i}^{2}$, for any $x \in B(p, 8 \varepsilon)$.

LEMma 3.12. There exists a positive constant c depending only on $n, k_{1}, \varepsilon$ such that for any $p \in X$ and any $\mu(p)<i \leq n,\left\|\nabla e_{i}^{p}\right\|_{\infty, 9 \varepsilon} \leq c$.

Proof. Let $x \in B(p, 9 \varepsilon)$ and consider $\gamma$ the minimizing geodesic from $p$ to $x$ of length $l(l<9 \varepsilon)$ and $\left\{X_{1}=\dot{\gamma}(t), X_{2}, \ldots, X_{n}\right\}$ an orthonormal basis of $E_{x}$ with $\nabla_{X_{i}} X_{j}=0$. Then

$$
\left|\nabla e_{i}^{p}(x)\right|^{2}=\sum_{j=1}^{n}\left|\nabla_{X_{j}} e_{i}^{p}(x)\right|^{2} \leq \sum_{j=1}^{n}\left(\int_{0}^{l}\left|\nabla_{\dot{\gamma}(t)} \nabla_{X_{j}} e_{i}^{p}(x)\right| d t\right)^{2}
$$

but $\left|R^{E}\left(\dot{\gamma}(t), X_{j}\right) e_{i}^{p}\right|=\left|\nabla_{\dot{\gamma}(t)} \nabla_{X_{j}} e_{i}^{p}\right| \leq k_{1}$. Therefore $\left|\nabla e_{i}^{p}(x)\right|^{2} \leq k_{1}^{2} l^{2} n$ and this concludes the proof.

3.2. Construction of the twisted Laplacian. The construction of $\Delta_{A}$ differs according to the assumptions done on $E$. However, the basic idea is the same in all cases and relies on the fact that $A$ has to express the holonomy. So let us consider $p, q \in X, p \in N(q)$ and let $x \in B(p, 8 \varepsilon) \cap B(q, 8 \varepsilon)$. Then define $a(p, q)_{i j}(x)$ by

$$
e_{j}^{p}(x)=\sum_{i=1}^{n} a(p, q)_{i j}(x) e_{i}^{q}(x) \quad \forall j=1, \ldots, n
$$

where $e_{i}^{p}, e_{j}^{q}$ are defined in Section 3.1. We define $A(p, q): \mathbb{R}^{n} \rightarrow \mathbb{R}^{n}$ on the canonical basis $\left\{e_{1}, \ldots e_{n}\right\}$ of $\mathbb{R}^{n}$ by $A(p, q) e_{j}=\sum_{i=1}^{n} A(p, q)_{i j} e_{i}$, where $A(p, q)_{i j}$ is defined as follows.

If $\mathbf{E}$ is of harmonic curvature then define $A(p, q)_{i j}$ by

$$
A(p, q)_{i j}=a(p, q)_{i j}(q) .
$$


If $\mathbf{E}$ is of complex (or quaternionic) rank one then define $A(p, q)_{i j}$ by

$$
A(p, q)_{i j}=\frac{1}{V_{p q}} \int_{B_{p q}} a(p, q)_{i j}(x) d V(x)
$$

where $B_{p q}$ is the ball centered at the mid-point of $p$ and $q$ of radius $5 \varepsilon$ and $V_{p q}$ denotes its volume. Note that $B_{p q} \supseteq B(p, 3 \varepsilon) \cup B(q, 3 \varepsilon)$.

REMARK 3.13. In the canonical basis of $\mathbb{R}^{n}$, we can write

$$
D_{A} f(p, q)=\sum_{i=1}^{n} D_{A} f(p, q)_{i} e_{i}=\sum_{i=1}^{n}\left(f_{i}(q)-\sum_{j=1}^{n} A(p, q)_{i j} f_{j}(p)\right) e_{i}
$$

REMARK 3.14. If $E$ is of harmonic curvature, we have by definition $e_{j}^{p}(q)=\sum_{i=1}^{n} A(p, q)_{i j} e_{i}^{q}(q), \forall j=1, \ldots, n$.

REMARK 3.15. If $E$ is flat, $a(p, q)_{i j}(x)$ is constant and so for $j=1, \ldots, n$ and for any $x \in B(p, 8 \varepsilon) \cap B(q, 8 \varepsilon), e_{j}^{p}(x)=\sum_{i=1}^{n} A(p, q)_{i j} e_{i}^{q}(x)$. Moreover, in this case $A(p, q) A(p, q)^{t}=I d$ and $A(p, q)^{t}=A(q, p)$. So that $\Delta_{A}$ is a discrete magnetic Laplacian.

If $\mathbf{E}$ is of harmonic curvature let $V: \mathcal{F}(X) \rightarrow \mathcal{F}(X)$ be defined by

$$
(V f)(p)=\sum_{i \leq \mu(p)} \lambda_{i}(p) f_{i}(p) e_{i}+\sum_{i>\mu(p)} f_{i}(p) e_{i}
$$

If $\mathbf{E}$ is of complex (or quaternionic) rank one let $V: \mathcal{F}(X) \rightarrow \mathcal{F}(X)$ be defined by

$$
(V f)(p)=\left(\lambda_{1}(p)+\sum_{q \in N(p)} \lambda_{1}(q)\right) f(p)
$$

REMARK 3.16. If the vector bundle is flat, then we have $V=0$.

\subsection{Smoothing operator.}

DeFinition 3.17. Let $\left\{\psi_{p}\right\}_{p \in X}$ be a partition of unity subordinate to the cover $\{B(p, 2 \varepsilon)\}_{p \in X}$. Define the smoothing operator $\mathcal{S}: \mathcal{F}(X) \rightarrow \Gamma(E)$ by

$$
(\mathcal{S} f)(x)=\sum_{p \in X} \psi_{p}(x)\left(\sum_{i=1}^{n} f_{i}(p) e_{i}^{p}(x)\right)
$$

where $f(p)=\sum_{i=1}^{n} f_{i}(p) e_{i}$.

Proposition 3.18. There exist constants $c_{0}, c_{1}, c_{2}$ and $\Lambda>0$ depending only on $m, n, k_{1}, k_{2}, \kappa$ and $\varepsilon$ such that

i) $\forall f \in \mathcal{F}(X),\|\mathcal{S} f\|^{2} \leq c_{0}\|f\|^{2}$,

ii) $\forall f \in \mathcal{F}(X),\|\nabla(\mathcal{S} f)\|^{2} \leq c_{1}\left(\left\|D_{A} f\right\|^{2}+(V f, f)\right)$,

iii) $\forall f \in \mathcal{F}(X)$ with $\left\|D_{A} f\right\|^{2}+(V f, f) \leq \Lambda\|f\|^{2},\|\mathcal{S} f\|^{2} \geq c_{2}\|f\|^{2}$ holds. 
Proof. For the first inequality note that $\{B(p, \varepsilon)\}_{p \in X}$ covers $M$. Hence

$$
\begin{aligned}
\|\mathcal{S} f\|^{2} & \leq\left.\sum_{q \in X} \int_{B(q, \varepsilon)} \sum_{p \in B(q, 3 \varepsilon) \cap X} \psi_{p}(x) \sum_{i=1}^{n} f_{i}(p) e_{i}^{p}(x)\right|^{2} d V(x) \\
& \leq\left(1+\delta^{\prime}\right) \sum_{q \in X} V(q, \varepsilon) \sum_{p \in B(q, 3 \varepsilon) \cap X}|f(p)|^{2} \leq\left(1+\delta^{\prime}\right) c\|f\|^{2} .
\end{aligned}
$$

In order to prove ii) fix $q \in X$ and let $x \in B(q, \varepsilon)$. Then as $\left\{\psi_{p}\right\}_{p \in X}$ is a partition of unity, we have $\sum_{p \in X} d \psi_{p}=0$, so that we can write

$$
\begin{aligned}
\nabla(\mathcal{S} f)(x)= & \sum_{p \in B(q, 3 \varepsilon) \cap X} \psi_{p}(x)\left(\sum_{i=1}^{n} f_{i}(p) \nabla e_{i}^{p}(x)\right)+ \\
& \sum_{p \in N(q)} d \psi_{p}(x)\left(\sum_{i=1}^{n} f_{i}(p) e_{i}^{p}(x)-\sum_{i=1}^{n} f_{i}(q) e_{i}^{q}(x)\right) .
\end{aligned}
$$

Then, Lemma 3.12 implies

$$
\begin{aligned}
\int_{B(q, \varepsilon)}\left|\sum_{p \in B(q, 3 \varepsilon) \cap X} \psi_{p}(x)\left(\sum_{i=1}^{n} f_{i}(p) \nabla e_{i}^{p}(x)\right)\right|^{2} d V(x) \leq \\
n \sum_{p \in B(q, 3 \varepsilon) \cap X}\left(\sum_{i \leq \mu(p)} f_{i}(p)^{2} \int_{B(q, \varepsilon)}\left|\nabla e_{i}^{p}(x)\right|^{2} d V(x)+c \sum_{i>\mu(p)} f_{i}(p)^{2}\right) \\
\leq c^{\prime} \sum_{p \in B(q, 3 \varepsilon) \cap X}(V f)(p) \cdot f(p) .
\end{aligned}
$$

To estimate the second term of (3.7), we need the following lemma.

Lemma 3.19. There exists a positive constant c depending only on $m, n, k_{1}, k_{2}$, $\kappa$ and $\varepsilon$ such that

$$
\begin{aligned}
\int_{B(q, \varepsilon)}\left|\sum_{i=1}^{n} f_{i}(p) e_{i}^{p}(x)-\sum_{i=1}^{n} f_{i}(q) e_{i}^{q}(x)\right|^{2} \leq \\
c\left(\left|D_{A} f(q, p)\right|^{2}+(V f)(p) \cdot f(p)+(V f)(q) \cdot f(q)\right) .
\end{aligned}
$$

Proof. See Appendix A.1. $\square$

Hence by (3.8), (3.7) and Lemma 3.19 we get

$$
\begin{aligned}
\int_{B(q, \varepsilon)}|\nabla(\mathcal{S} f)(x)|^{2} d V(x) \leq \\
c^{\prime \prime} \sum_{p \in B(q, 3 \varepsilon) \cap X}\left(\left|D_{A} f(q, p)\right|^{2}+(V f)(p) \cdot f(p)+(V f)(q) \cdot f(q)\right) .
\end{aligned}
$$

Then summing on $q \in X$ implies the claim. 
To prove the third part of Proposition 3.18, define $\left(\mathcal{S}_{q} f\right)(x)=\sum_{i=1}^{n} f_{i}(q) e_{i}^{q}(x)$ for $x$ in $B\left(q, \frac{\varepsilon}{2}\right)$. Then, by Lemma 3.19 we get

$$
\begin{aligned}
\int_{B\left(q, \frac{\varepsilon}{2}\right)} \mid(\mathcal{S} f)(x)- & \left.\left(\mathcal{S}_{q} f\right)(x)\right|^{2} d V(x)= \\
\int_{B\left(q, \frac{\varepsilon}{2}\right)} \mid & \left.\sum_{p \in N(q)} \psi_{p}(x) \sum_{j=1}^{n}\left(f_{j}(p) e_{j}^{p}(x)-f_{j}(q) e_{j}^{q}(x)\right)\right|^{2} d V(x) \leq \\
& c \sum_{p \in N(q)}\left(\left|D_{A} f(q, p)\right|^{2}+(V f)(p) \cdot f(p)+(V f)(q) \cdot f(q)\right) .
\end{aligned}
$$

As the balls of radius $\frac{\varepsilon}{2}$ centered on $X$ are disjoint, we can write

$$
\begin{aligned}
\|\mathcal{S} f\|^{2} \geq & \sum_{q \in X} \int_{B\left(q, \frac{\varepsilon}{2}\right)}\left|\left(\mathcal{S}_{q} f(x)-\mathcal{S} f(x)\right)-\mathcal{S}_{q} f(x)\right|^{2} d V(x) \\
\geq & \sum_{q \in X} \int_{B\left(q, \frac{\varepsilon}{2}\right)}\left|\mathcal{S}_{q} f(x)\right|^{2} d V(x) \\
& -2 \sum_{q \in X} \int_{B\left(q, \frac{\varepsilon}{2}\right)}\left|\mathcal{S}_{q} f(x)\right|\left|\mathcal{S} f(x)-\mathcal{S}_{q} f(x)\right| d V(x) .
\end{aligned}
$$

By construction, $\left(1-\delta^{\prime}\right)|f(q)|^{2} \leq\left|\mathcal{S}_{q} f(x)\right|^{2} \leq\left(1+\delta^{\prime}\right)|f(q)|^{2}$ and by Cauchy-Schwarz inequality combined with (3.9), we get

$$
\sum_{q \in X} \int_{B\left(q, \frac{\varepsilon}{2}\right)}\left|\mathcal{S}_{q} f(x)\right|\left|\mathcal{S} f(x)-\mathcal{S}_{q} f(x)\right| d V(x) \leq c^{\prime}\left(1+\delta^{\prime}\right)\|f\| \sqrt{\left\|D_{A} f\right\|^{2}+(V f, f)} .
$$

Hence, $\|\mathcal{S} f\|^{2} \geq\left(1-\delta^{\prime}\right) c^{\prime \prime}\|f\|^{2}-2 c^{\prime}\left(1+\delta^{\prime}\right)\|f\| \sqrt{\left\|D_{A} f\right\|^{2}+(V f, f)}$. Choose $\Lambda>0$ sufficiently small so that if $f$ satisfies $\left\|D_{A} f\right\|^{2}+(V f, f) \leq \Lambda\|f\|^{2}$, then

$$
\|\mathcal{S} f\|^{2} \geq\|f\|^{2}\left(\left(1-\delta^{\prime}\right) c^{\prime \prime}-2 c^{\prime}\left(1+\delta^{\prime}\right) \sqrt{\Lambda}\right) \geq \frac{\left(1-\delta^{\prime}\right) c^{\prime \prime}}{2}\|f\|^{2} .
$$

This concludes the proof of Proposition 3.18.

\subsection{Discretizing operator.}

Definition 3.20. Define the discretizing operator $\mathcal{D}: \Gamma(E) \rightarrow \mathcal{F}(X)$ by

$$
(\mathcal{D} s)(p)=\sum_{i=1}^{n} \frac{1}{V(p, 3 \varepsilon)} \int_{B(p, 3 \varepsilon)} s_{i}^{p}(x) d V(x) e_{i}
$$

where $s(x)=\sum_{i=1}^{n} s_{i}^{p}(x) e_{i}^{p}(x)$ for $x$ in $B(p, 3 \varepsilon)$.

Proposition 3.21. There exist constants $c_{0}^{\prime}, c_{1}^{\prime}, c_{2}^{\prime}$ and $\Lambda^{\prime}>0$ depending only on $m, n, \kappa, k_{1}, k_{2}$ and $\varepsilon$ such that 
i) $\forall s \in \Gamma(E)$, $\|\mathcal{D} s\|^{2} \leq c_{0}^{\prime}\|s\|^{2}$,

ii) $\forall s \in \Gamma(E),\left\|D_{A}(\mathcal{D} s)\right\|^{2}+(V(\mathcal{D} s), \mathcal{D} s) \leq c_{1}^{\prime}\|\nabla s\|^{2}$,

iii) $\forall s \in \Gamma(E)$ such that $\|\nabla s\|^{2} \leq \Lambda^{\prime}\|s\|^{2},\|\mathcal{D} s\|^{2} \geq c_{2}^{\prime}\|s\|^{2}$ holds.

Proof. The first point follows directly from the following inequality

$$
|\mathcal{D} s(p)|^{2} \leq c \int_{B(p, 3 \varepsilon)} \sum_{i=1}^{n}\left|s_{i}^{p}(x)\right|^{2} d V(x) \leq c\left(1-\delta^{\prime}\right)^{-1} \int_{B(p, 3 \varepsilon)}|s(x)|^{2} d V(x) .
$$

To prove the second point, we first prove that

$$
\left\|D_{A}(\mathcal{D} s)\right\|^{2}+(V(\mathcal{D} s), \mathcal{D} s) \leq c\left(\|\nabla s\|^{2}+\sum_{p \in X}(\widetilde{V} s)(p)\right)
$$

where if $\mathbf{E}$ is of harmonic curvature then

$$
(\widetilde{V} s)(p)=\left(\sum_{i \leq \mu(p)} \lambda_{i}(p) \int_{B(p, 3 \varepsilon)}\left|s_{i}^{p}\right|^{2} d V+\sum_{i>\mu(p)} \int_{B(p, 3 \varepsilon)}\left|s_{i}^{p}\right|^{2} d V\right)
$$

and if $\mathbf{E}$ is of complex (or quaternionic) rank one

$$
(\widetilde{V} s)(p)=\left(\lambda_{1}(p)+\sum_{q \in N(p)} \lambda_{1}(q)\right) \int_{B(p, 3 \varepsilon)}|s|^{2} d V
$$

and $s$ is written locally as $s(x)=\sum_{i=1}^{n} s_{i}^{p}(x) e_{i}^{p}(x)$ for $x \in B(p, 8 \varepsilon)$. First, $\left|(\mathcal{D} s)(p)_{j}\right|^{2} \leq c \int_{B(p, 3 \varepsilon)}\left|s_{j}^{p}(x)\right|^{2} d V(x)$ implies obviously

$$
(V(\mathcal{D} s), \mathcal{D} s) \leq \sum_{p \in X} c^{\prime}(\widetilde{V} s)(p)
$$

Secondly, for $p$ and $q \in N(p)$ let us introduce $B_{p q}^{\prime} \subseteq B(p, 3 \varepsilon) \cap B(q, 3 \varepsilon)$ the ball centered at the mid-point of $p$ and $q$ of radius $\varepsilon$ and $V_{p q}^{\prime}$ its volume. Then

$$
\begin{aligned}
& \left|D_{A}(\mathcal{D} s)(q, p)\right|^{2}= \\
& \sum_{i=1}^{n}\left(\frac{1}{V_{p q}^{\prime}} \int_{B_{p q}^{\prime}}\left|\mathcal{D} s(p)_{i}-\sum_{j=1}^{n} A(q, p)_{i j} \mathcal{D} s(q)_{j}\right| d V(y)\right)^{2} \\
\leq & 3 \sum_{i=1}^{n}\left(\frac{1}{V_{p q}^{\prime}} \int_{B_{p q}^{\prime}}\left|\mathcal{D} s(p)_{i}-s_{i}^{p}(y)\right| d V(y)\right)^{2} \\
+ & 3 \sum_{i=1}^{n}\left(\frac{1}{V_{p q}^{\prime}} \int_{B_{p q}^{\prime}}\left|\sum_{j=1}^{n} A(q, p)_{i j}\left(s_{j}^{q}(y)-\mathcal{D} s(q)_{j}\right)\right| d V(y)\right)^{2} \\
+ & 3 \sum_{i=1}^{n}\left(\frac{1}{V_{p q}^{\prime}} \int_{B_{p q}^{\prime}}\left|s_{i}^{p}(y)-\sum_{j=1}^{n} A(q, p)_{i j} s_{j}^{q}(y)\right| d V(y)\right)^{2} .
\end{aligned}
$$

We estimate each of the three terms separately. 
By a result of Kanai (see [10], Lemma VI.5.5), there exists $c_{K}>0$ depending only on $\varepsilon$ and $\kappa$ such that

$$
\frac{1}{V_{p q}^{\prime}} \int_{B_{p q}^{\prime}}\left|\mathcal{D} s(p)_{i}-s_{i}^{p}(y)\right| d V(y) \leq c_{K} \int_{B(p, 3 \varepsilon)}\left|d s_{i}^{p}(y)\right| d V(y) .
$$

Moreover

$$
\sqrt{1-\delta^{\prime}}\left|d s_{i}^{p}(y)\right| \leq\left|\sum_{j=1}^{n} d s_{j}^{p}(y) e_{j}^{p}(y)\right|=\left|\nabla s(y)-\sum_{j=1}^{n} s_{j}^{p}(y) \nabla e_{j}^{p}(y)\right| .
$$

Therefore

$$
\begin{aligned}
\sqrt{1-\delta^{\prime}} \int_{B(p, 3 \varepsilon)}\left|d s_{i}^{p}(y)\right| d V(y) \leq \\
\\
\quad\left(V(p, 3 \varepsilon) \int_{B(p, 3 \varepsilon)}|\nabla s(y)|^{2} d V(y)\right)^{\frac{1}{2}}+n \sum_{j=1}^{n}\left\|\nabla e_{j}^{p}\right\|_{2,3 \varepsilon}\left\|s_{j}^{p}\right\|_{2,3 \varepsilon}
\end{aligned}
$$

so that we obtain by Lemma 3.12 and by construction of $e_{j}^{p}$

$$
\sum_{i=1}^{n}\left(\int_{B(p, 3 \varepsilon)}\left|d s_{i}^{p}(y)\right| d V(y)\right)^{2} \leq c \int_{B(p, 3 \varepsilon)}|\nabla s(y)|^{2} d V(y)+c \widetilde{V} s(p) .
$$

We have then the following upper bound for (3.12)

$$
\begin{aligned}
& \sum_{i=1}^{n}\left(\frac{1}{V_{p q}^{\prime}} \int_{B_{p q}^{\prime}}\left|\mathcal{D} s(p)_{i}-s_{i}^{p}(y)\right|\right.d V(y))^{2} \\
& \leq c_{K}^{2} c\left(\int_{B(p, 3 \varepsilon)}|\nabla s(y)|^{2} d V(y)+(\widetilde{V} s)(p)\right) .
\end{aligned}
$$

By the same kind of arguments as for (3.12) and using that $\sum_{i, j=1}^{n}\left|A(q, p)_{i j}\right|^{2}$ is bounded above by a uniform constant, we can bound (3.13) as follows

$$
\begin{gathered}
\sum_{i=1}^{n}\left(\frac{1}{V_{p q}^{\prime}} \int_{B_{p q}^{\prime}}\left|\sum_{j=1}^{n} A(q, p)_{i j}\left(s_{j}^{q}(y)-\mathcal{D} s(q)_{j}\right)\right| d V(y)\right)^{2} \leq \\
c^{\prime}\left(\int_{B(q, 3 \varepsilon)}|\nabla s(y)|^{2} d V(y)+(\widetilde{V} s)(q)\right) .
\end{gathered}
$$

The last term (3.14) is then bounded by the following lemma

Lemma 3.22. There exists a positive constant c depending only on $m, n, k_{1}, k_{2}$, $\kappa$ and $\varepsilon$ such that

$$
\sum_{i=1}^{n}\left(\int_{B_{p q}^{\prime}}\left|s_{i}^{p}(y)-\sum_{j=1}^{n} A(q, p)_{i j} s_{j}^{q}(y)\right| d V(y)\right)^{2} \leq c((\widetilde{V} f)(p)+(\widetilde{V} f)(q)) .
$$


Proof. See Appendix A.2.

Finally, (3.16), (3.17) and Lemma 3.22 imply that

$$
\begin{aligned}
\left|D_{A}(\mathcal{D} s)(p, q)\right|^{2} \leq & c^{\prime \prime}\left(\int_{B(p, 3 \varepsilon)}|\nabla s(y)|^{2} d V(y)+\int_{B(q, 3 \varepsilon)}|\nabla s(y)|^{2} d V(y)\right) \\
& +c^{\prime \prime}((\widetilde{V} s)(p)+(\widetilde{V} s)(q)) .
\end{aligned}
$$

Taking the sum over $p$ and $q$ leads to

$$
\left\|D_{A}(\mathcal{D} s)\right\|^{2} \leq c^{\prime \prime \prime}\left(\|\nabla s\|^{2}+\sum_{p \in X}(\widetilde{V} s)(p)\right)
$$

so that (3.18),(3.11) imply (3.10). In order to conclude the proof of point $i i)$ of this lemma, we have to show that there exists $c>0$ such that

$$
\sum_{p \in X}(\widetilde{V} s)(p) \leq c\|\nabla s\|^{2}
$$

Fix $q \in X$, let $B=B(q, 10 \varepsilon), V(B)$ its volume. Let $(\cdot, \cdot)_{B}$ and $\|\cdot\|_{B}$ the $L^{2}$-inner product respectively the $L^{2}$-norm on $E$ restricted to $B$. We are going to show that there exists $c>0$ such that

$$
(\widetilde{V} s)(q) \leq c \sum_{p \in B(q, 3 \varepsilon) \cap X}\|\nabla s\|_{B(p, 10 \varepsilon)}^{2} .
$$

Then (3.19) is a direct consequence of (3.20). To prove (3.20) we have to consider separately the cases $E$ is of complex (or quaternionic) rank one and $E$ is of harmonic curvature.

Assume E is of rank one. The proof of (3.20) in this case is much easier than in the other case as the potential involves only the first eigenvalue of the ball. Recall that $\lambda_{1}(q) \leq \frac{\|\nabla s\|_{B}^{2}}{\|s\|_{B}^{2}}$ for any non-zero $s$. Therefore and as $B(q, 3 \varepsilon) \subseteq B(p, 10 \varepsilon)$ for any $p \in N(q)$

$$
(\widetilde{V} s)(q) \leq\|s\|_{B(q, 3 \varepsilon)}^{2} \sum_{p \in B(q, 3 \varepsilon) \cap X} \frac{\|\nabla s\|_{B(p, 10 \varepsilon)}^{2}}{\|s\|_{B(p, 10 \varepsilon)}^{2}} \leq \sum_{p \in B(q, 3 \varepsilon) \cap X}\|\nabla s\|_{B(p, 10 \varepsilon)}^{2}
$$

and this concludes the first case.

Assume $\mathbf{E}$ is of harmonic curvature. If $y \in B$, write $s(y)$ as a sum of orthogonal sections (with respect to $\left.(\cdot, \cdot)_{B}\right) s(y)=\widetilde{s}(y)+s^{\perp}(y)$ with $\widetilde{s}(y)=$ $\sum_{j \leq \mu(q)} \frac{\left(s, e_{j}^{q}\right)_{B}}{V(B)} e_{j}^{q}(y)$. We have the following properties of the decomposition.

$$
\begin{aligned}
\left(s^{\perp}, e_{j}^{q}\right)_{B} & =0, \forall j \leq \mu(q), & \left(\nabla s^{\perp}, \nabla \widetilde{s}\right)_{B} & =0, \\
\|s\|_{B}^{2} & =\left\|s^{\perp}\right\|_{B}^{2}+\|\widetilde{s}\|_{B}^{2}, & \|\nabla s\|_{B}^{2} & =\left\|\nabla s^{\perp}\right\|_{B}^{2}+\|\nabla \widetilde{s}\|_{B}^{2}, \\
\|\widetilde{s}\|_{B}^{2} & =\sum_{j \leq \mu(q)} \frac{\left(s, e_{j}^{q}\right)_{B}^{2}}{V(B)}, & \|\nabla \widetilde{s}\|_{B}^{2} & =\sum_{j \leq \mu(q)} \frac{\left(s, e_{j}^{q}\right)_{B}^{2}}{V(B)} \lambda_{j}(q) .
\end{aligned}
$$


Consider then two cases. First assume $\left\|s^{\perp}\right\|_{B}^{2}=0$. Then $s(y)=\widetilde{s}(y)$ which means that if $y \in B(p, 10 \varepsilon)$

$$
s_{j}^{q}(y)= \begin{cases}0 & \text { if } j>\mu(q) \\ \frac{\left(s, e_{j}^{q}\right)_{B}}{V(B)} & \text { if } j \leq \mu(q) .\end{cases}
$$

Therefore

$$
\begin{aligned}
(\widetilde{V} s)(q) & =\left(\sum_{j \leq \mu(q)} \lambda_{j}(q) \int_{B(q, 3 \varepsilon)}\left|s_{j}^{q}\right|^{2} d V+\sum_{j>\mu(q)} \int_{B(q, 3 \varepsilon)}\left|s_{j}^{q}\right|^{2} d V\right) \\
& =V(q, 3 \varepsilon) \sum_{j \leq \mu(q)} \frac{\left(s, e_{j}^{q}\right)_{B}^{2}}{V(B)^{2}} \lambda_{j}(q) \leq c\|\nabla \widetilde{s}\|_{B}^{2} .
\end{aligned}
$$

Moreover as $s^{\perp}$ is zero $\|\nabla \widetilde{s}\|_{B}^{2}=\|\nabla s\|_{B}^{2}$ and so in this case (3.20) is verified.

For the second case, assume $\left\|s^{\perp}\right\|_{B}^{2} \neq 0$. Then apply max-min theorem to $s^{\perp}$ to obtain $\lambda_{\mu(q)+1}(q) \leq \frac{\left\|\nabla s^{\perp}\right\|_{B}^{2}}{\left\|s^{\perp}\right\|_{B}^{2}}$ and by definition of $\mu(q)$ this implies that

$$
\delta\left\|s^{\perp}\right\|_{B}^{2} \leq\left\|\nabla s^{\perp}\right\|_{B}^{2} .
$$

Moreover, let us rewrite $s^{\perp}$ as follows, for $y \in B(q, 8 \varepsilon)$

$$
s^{\perp}(y)=\sum_{j \leq \mu(q)}\left(s_{j}^{q}(y)-\frac{\left(s, e_{j}^{q}\right)_{B}}{V(B)}\right) e_{j}^{q}(y)+\sum_{j>\mu(q)} s_{j}^{q}(y) e_{j}^{q}(y) .
$$

As $\left\{e_{j}^{q}(y)\right\}$ is an almost orthonormal basis, we obtain for $y \in B(q, 8 \varepsilon)$

$$
\sum_{j \leq \mu(q)}\left|s_{j}^{q}(y)-\frac{\left(s, e_{j}^{q}\right)_{B}}{V(B)}\right|^{2}+\sum_{j>\mu(q)}\left|s_{j}^{q}(y)\right|^{2} \leq\left(1-\delta^{\prime}\right)^{-1}\left|s^{\perp}(y)\right|^{2} .
$$

In particular, this implies

$$
\sum_{j>\mu(q)} \int_{B(q, 3 \varepsilon)}\left|s_{j}^{q}(y)\right|^{2} d V(y) \leq\left(1-\delta^{\prime}\right)^{-1}\left\|s^{\perp}\right\|_{B}^{2}
$$

and

$$
\begin{array}{rl}
\sum_{j \leq \mu(q)} \lambda_{j}(q) \int_{B(q, 3 \varepsilon)}\left|s_{j}^{q}(y)\right|^{2} d V(y) \leq & \\
2 \sum_{j \leq \mu(q)} \lambda_{j}(q) \int_{B(q, 3 \varepsilon)}\left|s_{j}^{q}(y)-\frac{\left(s, e_{j}^{q}\right)_{B}}{V(B)}\right|^{2} & d V(y)+2 \sum_{j \leq \mu(q)} \frac{\left(s, e_{j}^{q}\right)_{B}^{2}}{V(B)} \lambda_{j}(q) \\
& \leq \frac{2 \delta}{1-\delta}\left\|s^{\perp}\right\|_{B}^{2}+2\|\nabla \widetilde{s}\|_{B}^{2} .
\end{array}
$$

Then (3.22) and (3.23) imply that $(\widetilde{V} s)(q) \leq c\left(\left\|s^{\perp}\right\|_{B}^{2}+\|\nabla \widetilde{s}\|_{B}^{2}\right)$. Use (3.21) together with this inequality to obtain (3.20) and therefore (3.19). Finally (3.10) together with (3.19) imply $i$ i). 
To prove iii) consider the following sum. By the work of Buser (Lemma 5.1 in [8]), there exists $c_{B}>0$ depending only on $m, \kappa$ and $\varepsilon$ such that

$$
\sum_{i=1}^{n} \int_{B(p, 3 \varepsilon)}\left|\mathcal{D} s(p)_{i}-s_{i}^{p}(x)\right|^{2} d V(x) \leq c_{B} \sum_{i=1}^{n} \int_{B(p, 3 \varepsilon)}\left|d s_{i}^{p}(x)\right|^{2} d V(x) .
$$

Moreover, using (3.15) we obtain

$$
\begin{aligned}
& \sum_{i=1}^{n} \int_{B(p, 3 \varepsilon)}\left|\mathcal{D} s(p)_{i}-s_{i}^{p}(x)\right|^{2} d V(x) \leq \\
& \quad \frac{2 n c_{B}}{1-\delta^{\prime}}\left(\int_{B(p, 3 \varepsilon)}|\nabla s(y)|^{2} d V(y)+n \sum_{j=1}^{n}\left\|\nabla e_{j}^{p}\right\|_{\infty, 3 \varepsilon}^{2}\left\|s_{j}^{p}(y)\right\|_{2,3 \varepsilon}^{2}\right) .
\end{aligned}
$$

Therefore, from (3.24) we obtain

$$
\begin{aligned}
&|\mathcal{D} s(p)|^{2} \geq c \int_{B(p, 3 \varepsilon)} \sum_{i=1}^{n}\left|\left(s_{i}^{p}(x)-\mathcal{D} s(p)_{i}\right)-s_{i}^{p}(x)\right|^{2} d V(x) \\
& \geq c \int_{B(p, 3 \varepsilon)} \sum_{i=1}^{n}\left|s_{i}^{p}(x)\right|^{2} d V(x)-2 c \int_{B(p, 3 \varepsilon)^{i=1}}^{n}\left|s_{i}^{p}(x) \| \mathcal{D} s(p)_{i}-s_{i}^{p}(x)\right| d V(x) \\
& \geq c^{\prime}\|s\|_{B(p, 3 \varepsilon)}^{2}-c^{\prime \prime}\|s\|_{B(p, 3 \varepsilon)}\left(\|\nabla s\|_{B(p, 3 \varepsilon)}^{2}+\sum_{j=1}^{n}\left\|\nabla e_{j}^{p}\right\|_{\infty, 3 \varepsilon}^{2}\left\|s_{j}^{p}\right\|_{2,3 \varepsilon}^{2}\right)^{\frac{1}{2}} .
\end{aligned}
$$

Assume $\mathbf{E}$ is of harmonic curvature and combine Lemma 3.3 and Lemma 3.12 with (3.25) to obtain

$$
|\mathcal{D} s(p)|^{2} \geq c^{\prime}\|s\|_{B(p, 3 \varepsilon)}^{2}-c^{\prime \prime}\|s\|_{B(p, 3 \varepsilon)}\left(\|\nabla s\|_{B(p, 3 \varepsilon)}^{2}+(\widetilde{V} s)(p)\right)^{\frac{1}{2}} .
$$

Moreover, by (3.20) $(\widetilde{V} s)(p)$ is bounded above by $c \sum_{q \in B(p, 3 \varepsilon) \cap X}\|\nabla s\|_{B(q, 10 \varepsilon)}^{2}$. Then, taking the sum over $p \in X$ produces new $c^{\prime}, c^{\prime \prime}>0$ such that

$$
\|\mathcal{D} s\|^{2} \geq c^{\prime}\|s\|^{2}-c^{\prime \prime}\|s\|\|\nabla s\| \text {. }
$$

Finally, if $\|\nabla s\|^{2} \leq \Lambda^{\prime}\|s\|^{2}$, we get $\|\mathcal{D} s\|^{2} \geq\|s\|^{2}\left(c^{\prime}-c^{\prime \prime} \sqrt{\Lambda^{\prime}}\right)$. Choose then $\Lambda^{\prime}$ suitably to conclude the proof of the proposition in this case.

Assume $\mathbf{E}$ is of rank one. If $\lambda_{1}(p) \leq \delta$, by Lemma 3.3, $\left\|\nabla e_{j}^{p}\right\|_{\infty, 3 \varepsilon}^{2} \leq c \lambda_{1}^{s}(p)$. If $\lambda_{1}(p)>\delta$, by Lemma $3.12\left\|\nabla e_{j}^{p}\right\|_{\infty, 3 \varepsilon}^{2} \leq c \leq c \delta^{-1} \lambda_{1}(p)$. Therefore, (3.25) can be changed in (with new constants $c, c^{\prime}, c^{\prime \prime}$ )

$$
|\mathcal{D} s(p)|^{2} \geq \begin{cases}\left(c^{\prime}-c \lambda_{1}^{\frac{s}{2}}(p)\right)\|s\|_{B(p, 3 \varepsilon)}^{2}-c^{\prime \prime}\|s\|_{B(p, 3 \varepsilon)}\|\nabla s\|_{B(p, 3 \varepsilon)} & \text { if } \lambda_{1}(p) \leq \delta \\ c^{\prime}\|s\|_{B(p, 3 \varepsilon)}^{2}-c^{\prime \prime}\|s\|_{B(p, 3 \varepsilon)}\|\nabla s\|_{B(p, 10 \varepsilon)} & \text { otherwise. }\end{cases}
$$


By choosing $\delta$ smaller, we can assume that if $\lambda_{1}(p) \leq \delta, c^{\prime}-c \lambda_{1}(p)^{\frac{s}{2}} \geq c^{\prime \prime \prime}>0$. This implies that (for any values of $\lambda_{1}(p)$ )

$$
|\mathcal{D} s(p)|^{2} \geq c^{\prime \prime \prime}\|s\|_{B(p, 3 \varepsilon)}^{2}-c^{\prime \prime}\|\nabla s\|_{B(p, 3 \varepsilon)}\|s\|_{B(p, 10 \varepsilon)} .
$$

Then, take the sum over $p \in X$ to obtain for $\|\nabla s\| \leq \Lambda^{\prime}\|s\|$

$$
\|\mathcal{D} s\|^{2} \geq c^{\prime \prime \prime}\|s\|^{2}-c^{\prime \prime}\|\nabla s\|\|s\| \geq\|s\|^{2}\left(c^{\prime \prime \prime}-c^{\prime \prime} \sqrt{\Lambda^{\prime}}\right)
$$

and conclude choosing $\Lambda^{\prime}$ suitably.

\subsection{Upper bounds.}

Lemma 3.23. Let $m, n, k_{1}, k_{2}, \kappa, r_{0}, \varepsilon$ be as before. Then there exist positive constants $c_{3}$ and $c_{3}^{\prime}$ depending only on $m, n, k_{1}, k_{2}, \kappa$, $\varepsilon$ so that for any vector bundle $E \in \mathcal{E}\left(n, k_{1}, k_{2}\right)$ over any $M \in \mathcal{M}\left(m, \kappa, r_{0}\right)$, for any $X \varepsilon$-discretization of $E$ and for $\Delta_{A}+V$ constructed in Section 3.2, we have

i) $\lambda_{k}(E) \leq c_{3}, \forall k \leq n|X|$,

ii) $\lambda_{k}(X, \bar{A}, V) \leq c_{3}^{\prime}, \forall k \leq n|X|$.

Proof. $i$ ) Let $p_{i}$ be a vertex of $X$ and consider $f_{i}: M \rightarrow \mathbb{R}$ the first eigenfunction of the Dirichlet problem for the ball centered at $p_{i}$ of radius $\frac{\varepsilon}{2}$ extended by zero. By Cheng's comparison theorem $\frac{\left\|d f_{i}\right\|^{2}}{\left\|f_{i}\right\|^{2}} \leq \lambda_{1}\left(\frac{\varepsilon}{2}, \kappa\right)$ (where $\lambda_{1}\left(\frac{\varepsilon}{2}, \kappa\right)$ denotes the first non-zero eigenvalue of the Dirichlet problem on the ball of radius $\frac{\varepsilon}{2}$ in the simply connected space of constant sectional curvature $-\kappa$ and of same dimension as $M$ ). Define then the sections $\sigma_{j}^{i}(x)=f_{i}(x) e_{j}^{p_{i}}(x)$ for $1 \leq i \leq|X|$, and $1 \leq j \leq n$. Then $\left\{\sigma_{j}^{i}|1 \leq i \leq| X \mid, 1 \leq j \leq n\right\}$ spans a vector subset $W$ of $\Gamma(E)$ of dimension $n|X|$ as $\left\{e_{j}^{p_{i}}\right\}_{j=1, \ldots, n}$ is an almost orthonormal frame. Moreover

$$
\nabla \sigma_{j}^{i}(x)=d f_{i}(x) e_{j}^{p_{i}}(x)+f_{i}(x) \nabla e_{j}^{p_{i}}(x)
$$

hence by construction of $e_{j}^{p_{i}}$ and Lemma 3.3 and Lemma 3.12, we have

$$
\left\|\nabla \sigma_{j}^{i}\right\|^{2} \leq c\left(\left\|d f_{i}\right\|^{2}+\left\|f_{i}\right\|^{2}\right)
$$

so that by definition of the $f_{i}$ 's

$$
\left\|\nabla \sigma_{j}^{i}\right\|^{2} \leq c\left\|f_{i}\right\|^{2}\left(1+\lambda_{1}\left(\frac{\varepsilon}{2}, \kappa\right)\right) .
$$

By min-max theorem we get then

$$
\lambda_{k}(E) \leq c^{\prime} \max \left\{\frac{\sum_{i, j} a_{i j}^{2}\left\|\nabla \sigma_{j}^{i}\right\|^{2}}{\sum_{i, j} a_{i j}^{2}\left\|\sigma_{j}^{i}\right\|^{2}}\right\} \leq c^{\prime} c\left(1+\lambda_{1}\left(\frac{\varepsilon}{2}, \kappa\right)\right) .
$$

This concludes the first part of the lemma.

ii) Let $f \in \mathcal{F}(X)$. As $A(p, q)$ is a change of almost orthonormal bases we have

$$
\begin{aligned}
\left\|D_{A} f\right\|^{2}+(V f, f) & =\frac{1}{2} \sum_{p \in X} \sum_{q \in N(p)}|f(q)-A(p, q) f(p)|^{2}+\sum_{p \in X}(V f)(p) \cdot f(p) \\
& \leq c \sum_{p \in X} \sum_{q \in N(p)}\left(|f(p)|^{2}+|f(q)|^{2}\right)+\max \{\delta, 1\}\|f\|^{2} \\
& \leq\left(2 c \nu_{X}+\max \{\delta, 1\}\right)\|f\|^{2} .
\end{aligned}
$$

Therefore, $R(f) \leq 2 c \nu_{X}+\max \{\delta, 1\}, \forall f \in \mathcal{F}(X) \backslash\{0\}$ and this implies $\lambda_{k}(X, A, V) \leq$ $2 c \nu_{X}+\max \{\delta, 1\}, \forall k \leq n|X|$. $\square$ 
3.6. Conclusion. Proof of Theorem 3.1: by symmetry of the results concerning the smoothing and the discretizing, it suffices to deduce $\lambda_{k}(E) \leq c \lambda_{k}(X, A, V)$. The proof proceeds in two steps.

First, assume that $k$ is such that $\lambda_{k}(X, A, V) \geq \Lambda$, for $\Lambda$ given by Proposition 3.18 iii). Then, Lemma $3.23 i$ ) leads to $\lambda_{k}(E) \leq c_{3} \Lambda^{-1} \lambda_{k}(X, A, V)$. This is the required inequality.

Secondly, assume that $k$ is such that $\lambda_{k}(X, A, V) \leq \Lambda$. Let $W_{k}$ be the $k$ dimensional vector subspace of $\mathcal{F}(X)$ spanned by $f_{i}: X \rightarrow \mathbb{R}^{n}, i=1, \ldots, k$, $\lambda_{i}(X, A, V)$-eigenfunction of $\Delta_{A}$ chosen so that $\left(f_{i}, f_{j}\right)=\delta_{i j}|X|$. By min-max theorem, $\lambda_{k}(X, A, V)=\max \left\{R(f): f \in W_{k} \backslash\{0\}\right\}$. Let then $\mathcal{S} W_{k}$ be the vector subspace of $\Gamma(E)$ spanned by the $\mathcal{S} f_{i}$ 's i.e. $\mathcal{S} W_{k}=\left\langle\mathcal{S} f_{1}, \ldots, \mathcal{S} f_{k}\right\rangle=\left\{\mathcal{S} f \mid f \in W_{k} \backslash\{0\}\right\}$. As $\lambda_{k}(X, A, V) \leq \Lambda$, for any non-zero function $f$ in $W_{k}$, we have $\left\|D_{A} f\right\|^{2}+(V f, f) \leq$ $\Lambda\|f\|^{2}$. Hence, by Proposition 3.18 iii), for any $f$ in $W_{k},\|\mathcal{S} f\|^{2} \geq c_{2}\|f\|^{2}$ holds. In particular, $\mathcal{S} f$ is the zero function if and only if $f$ is zero which means that $\mathcal{S} W_{k}$ is $k$-dimensional. So we can apply min-max theorem to $\mathcal{S} W_{k}$ and obtain

$$
\lambda_{k}(E) \leq \max \left\{R(\mathcal{S} f) \mid f \in W_{k} \backslash\{0\}\right\} .
$$

Moreover, by Proposition $3.18 \mathrm{ii}$ ) and $\mathrm{iii}$ ) we obtain that $R(\mathcal{S} f) \leq \frac{c_{1}}{c_{2}} R(f)$ for any non-zero $f$ in $W_{k}$, which leads to

$$
\lambda_{k}(E) \leq \frac{c_{1}}{c_{2}} \max \left\{R(f) \mid f \in W_{k} \backslash\{0\}\right\}=\frac{c_{1}}{c_{2}} \lambda_{k}(X, A, V) .
$$

This concludes the proof.

4. Estimation of the first non-zero eigenvalue for a flat vector bundle. Let $\left(E^{n}, \nabla\right)$ be a flat Riemannian vector bundle with irreducible holonomy over $M \in$ $\mathcal{M}\left(m, \kappa, r_{0}\right)$. We recall the definition of the constant related to the holonomy given by Ballmann, Brüning and Carron in [2]. If $c$ is a unit speed loop, denote by $H_{c}$ its holonomy. Then there exists $\alpha>0$ such that $\forall x \in M, \forall v \in E_{x}$ there exists a smooth unit speed loop $c_{x, v}$ of length less than two diameters of $M$ such that

$$
\left|H_{c_{x, v}}(v)-v\right| \geq \alpha|v| .
$$

The following theorem shows that if $E$ has significant holonomy, then the first eigenvalue of $\bar{\Delta}$ can not be too small. Conversely, if there exists $v$ in $E_{x}$ which has a small holonomy, then the first eigenvalue is not too large.

TheOREM 4.1. Let $\left(E^{n}, \nabla\right)$ be a flat Riemannian vector bundle over $M \in$ $\mathcal{M}\left(m, \kappa, r_{0}\right)$ with irreducible holonomy. Then there exist $c, c^{\prime}>0$ depending only on $m, n, \kappa, r_{0}$ such that

$$
\lambda_{1}(E) \geq c^{\prime} \frac{\alpha^{2}}{d(M)^{2} c^{d(M)}}
$$

where $d(M)$ denotes the diameter of $M$.

Moreover, if there exist $p_{0} \in M, v_{0} \in E_{p_{0}}$ and $\alpha^{\prime}$ such that for any loop $c$ at $p_{0}$ of length less than $7 d(M),\left|H_{c}\left(v_{0}\right)-v_{0}\right| \leq \alpha^{\prime}\left|v_{0}\right|$ then, there exists $c^{\prime \prime}>0$ depending only on $n, m, \kappa$ and $r_{0}$ such that

$$
\lambda_{1}(E) \leq c^{\prime \prime} \alpha^{\prime 2}
$$


The first part of the theorem is in fact due to Ballmann, Brüning and Carron (see [2]). We present here a more conceptual proof that relies on the fact that the discrete magnetic Laplacian associated to a discretization of a flat bundle is strongly related to the holonomy of the vector bundle.

Proof. Let $\varepsilon=\frac{1}{100} r_{0}$ and let $X$ be an $\varepsilon$-discretization of $E$. Then by Theorem 3.1 there exist $\Delta_{A}$ a discrete magnetic Laplacian and $c>0$ such that $\lambda_{1}(E) \geq c \lambda_{1}(X, A)$. So it suffices to prove the statement for $\lambda_{1}(X, A)$. Let $f \in \mathcal{F}(X)$ such that $\Delta_{A} f=\lambda f$. Let $p_{0} \in X$ and $v_{0}=\sum_{i=1}^{n} f_{i}\left(p_{0}\right) e_{i}^{p_{0}} \in E_{p_{0}}$. By (4.1), there exists a smooth unit speed loop $c_{0}:[0, l] \rightarrow M$ at $p_{0}$ of length $l \leq 2 d(M)$ and $\left|H_{c_{0}}\left(v_{0}\right)-v_{0}\right| \geq \alpha\left|v_{0}\right|$. Let $N \in \mathbb{N}$ such that $N \frac{\varepsilon}{2} \leq l<(N+1) \frac{\varepsilon}{2}$ and consider a partition of $[0, l], 0=$ $t_{0}<t_{1}<\ldots<t_{N-1}<t_{N}=l$ such that $\frac{\varepsilon}{2} \leq t_{j}-t_{j-1} \leq \varepsilon$. By definition of $X$, $\forall j=1, \ldots, N-1, \exists p_{j} \in X$ such that $d\left(p_{j}, c_{0}\left(t_{j}\right)\right)<\varepsilon$. Moreover, let $p_{N}=p_{0} \in X$. Note that $d\left(p_{j-1}, p_{j}\right)<3 \varepsilon$. Consider then the piecewise geodesic loop $\bar{c}_{0}$ at $p_{0}$ passing through all $p_{j}, j=1, \ldots N-1$ (i.e $\bar{c}_{0}$ joins $p_{j-1}$ to $p_{j}$ via the minimizing geodesic $\left.p_{j-1} p_{j}\right)$. Note that $\bar{c}_{0}$ is of length less than $3 N \varepsilon \leq 12 d(M)$. Moreover, as $E$ is flat, the holonomy of $c_{0}$ is the same as the holonomy of $\bar{c}_{0}$. More precisely, parallel translation from $c_{0}\left(t_{j-1}\right)$ to $c_{0}\left(t_{j}\right)$ along $c_{0}$ is the same as parallel translation along minimizing geodesics from $c_{0}\left(t_{j-1}\right)$ to $p_{j-1}$, then from $p_{j-1}$ to $p_{j}$ and finally from $p_{j}$ to $c_{0}\left(t_{j}\right)$. Hence $H_{c_{0}}(v)=H_{\bar{c}_{0}}(v)$ for any $v \in E_{p_{0}}$. So that we obtain

$$
\left|H_{\bar{c}_{0}}\left(v_{0}\right)-v_{0}\right| \geq \alpha\left|v_{0}\right|=\alpha\left|f\left(p_{0}\right)\right| .
$$

Consider then $v_{j}=\sum_{i=1}^{n} f_{i}\left(p_{j}\right) e_{i}^{p_{j}} \in E_{p_{j}}$. By triangle inequality and as parallel transport is an isometry, we obtain easily the following inequality

$$
\alpha\left|f\left(p_{0}\right)\right| \leq \sum_{j=1}^{N}\left|\tau_{p_{j}, p_{j-1}} v_{j-1}-v_{j}\right| .
$$

Moreover, by construction of $D_{A}$ we have

$$
\begin{aligned}
\left|\tau_{p_{j}, p_{j-1}} v_{j-1}-v_{j}\right| & =\left|\sum_{i=1}^{n} f_{i}\left(p_{j-1}\right) \tau_{p_{j}, p_{j-1}} e_{i}^{p_{j-1}}-\sum_{i=1}^{n} f_{i}\left(p_{j}\right) e_{i}^{p_{j}}\right| \\
& =\left|\sum_{i=1}^{n}\left(\sum_{k=1}^{n} A\left(p_{j-1}, p_{j}\right)_{i k} f_{k}\left(p_{j-1}\right)-f_{i}\left(p_{j}\right)\right) e_{i}^{p_{j}}\right| \\
& =\left|D_{A} f\left(p_{j-1}, p_{j}\right)\right| .
\end{aligned}
$$

This implies that $\alpha\left|f\left(p_{0}\right)\right| \leq\left|D_{A} f\left(p_{0}, p_{1}\right)\right|+\ldots+\left|D_{A} f\left(p_{N-1}, p_{N}\right)\right|$. We have shown that for any $p_{0} \in X$, there exists a piecewise geodesic loop $\bar{c}_{0}=\left\{p_{0}, p_{1}, \ldots, p_{N}\right\}$ of length less than $12 d(M)$ such that

$$
\alpha^{2}\left|f\left(p_{0}\right)\right|^{2} \leq 4 \frac{d(M)}{\varepsilon}\left(\left|D_{A} f\left(p_{0}, p_{1}\right)\right|^{2}+\ldots+\left|D_{A} f\left(p_{N-1}, p_{N}\right)\right|^{2}\right)
$$

and $d\left(p_{j-1}, p_{j}\right)<3 \varepsilon$. The goal is to apply this last inequality to $\|f\|^{2}$. To that end, we need to find an upper bound for the number of loops of the kind $\{p, q, \ldots, p\}$ that can pass through $p \in X$ and $q \in N(p)$ and of length less than $12 d(M)$. This upper bound on the length of the loop implies that such a loop can pass through at most $P \leq 12 \frac{d(M)}{\varepsilon}$ points of $X$. Therefore, there are at most $\nu^{P-1}$ loops of the kind 
$\{p, q, \ldots, p\}$ and each of these loops is suitable for $P$ points in $X$. Hence, we obtain

$$
\begin{aligned}
\alpha^{2}\|f\|^{2} & \leq P \nu^{P-1} 8 \frac{d(M)}{\varepsilon}\left\|D_{A} f\right\|^{2} \\
& \leq 72 \frac{d(M)^{2}}{\varepsilon^{2}} \nu^{12 \frac{d(M)}{\varepsilon}}\left\|D_{A} f\right\|^{2} .
\end{aligned}
$$

This leads then to the conclusion of the first part $\alpha^{2} \frac{\varepsilon^{2}}{72 d(M)^{2} \nu^{12 \frac{d(M)}{\varepsilon}}} \leq \lambda$.

To prove the second part of the theorem let $\varepsilon=\frac{1}{100} r_{0}$ and $X$ be an $\varepsilon$-discretization of $E$ such that $p_{0} \in X$. Recall that $X$ is the set of vertices of a finite connected graph $G$. Then construct a spanning tree $S$ of $G$ (see [4], Section I.2) as follows. Let $X_{i}=\left\{p \in X \mid d_{G}\left(p, p_{0}\right)=i\right\}$ where $d_{G}$ denotes the path metric on $G$. Note that if $q$ is in $X_{i}$ then there exists $q^{\prime}$ in $X_{i-1}$ which is joined by an edge to $q$. Let then $S$ be the subgraph of $G$ with vertices set $X$ and edges set $E(S)=\left\{q q^{\prime} \mid q \neq p_{0}\right\}$. We have constructed a spanning tree $S$ of $G$.

By construction of $S$, for any $p$ in $X$ there exists a unique curve $c_{p}$ in $S$ joining $p$ to $p_{0}$ (i.e. $c_{p}$ is a piecewise geodesic curve $\left\{p, \ldots, p_{0}\right\}$ such that two consecutive points of $X$ in $c_{p}$ are joined in $S$ ). Moreover the length of such a $c_{p}$ is bounded above by $3 d(M)$. Now, choose in $E_{p_{0}}$ an orthonormal basis $\left\{e_{1}^{p_{0}}, \ldots, e_{n}^{p_{0}}\right\}$ and define an orthonormal basis $\mathcal{B}_{p}$ of $E_{p}$ by $\mathcal{B}_{p}=\left\{e_{i}^{p}=\tau_{c_{p}} e_{i}^{p_{0}}\right\}_{i=1, \ldots, n}$, where $\tau_{c_{p}}$ denotes parallel transport along $c_{p}$ from $p_{0}$ to $p$. Then $e_{i}^{p}(x)=\tau_{x, p} e_{i}^{p}$ gives a local orthonormal frame made of parallel sections. Hence, consider the discrete magnetic Laplacian $\Delta_{A}$ associated to this choice of bases (constructed as in Section 3.2) which satisfies $\lambda_{1}(E) \leq c \lambda_{1}(X, A)$ by Theorem 3.1. So that it suffices to prove the result for the first eigenvalue of $\Delta_{A}$. By min-max theorem $\lambda_{1}(X, A) \leq R(f)$ for any non-zero function on $X$. So consider $f: X \rightarrow \mathbb{R}^{n}$ defined by $f(p)=\sum_{i=1}^{n} v_{i} e_{i}$ where the $v_{i}$ 's are the coordinates of $v_{0}$ in the basis $\mathcal{B}_{p_{0}}$. If $p$ and $q$ are neighboring points in $X$ such that $d\left(p, p_{0}\right) \leq d\left(q, p_{0}\right)$ and $p \in c_{q}$, then we have $\tau_{q, p} e_{j}^{p}=e_{j}^{q}$. Hence in this case $A(p, q)_{i j}=\delta_{i j}$ and so $D_{A} f(p, q)=0$. In the other case i.e. if $p \in N(q), d\left(p, p_{0}\right) \leq d\left(q, p_{0}\right)$ and $p$ is not on $c_{q}$, consider the loop $c$ at $x_{0}$ going from $x_{0}$ to $p$ via $c_{p}$, from $p$ to $q$ via the minimizing geodesic $p q$ and from $q$ to $x_{0}$ via $c_{q}^{-1}$. Then $c$ is of length less than $7 d(M)$ and by assumption

$$
\left|H_{c}\left(v_{0}\right)-v_{0}\right| \leq \alpha^{\prime}\left|v_{0}\right|
$$

But, we have $H_{c}\left(v_{0}\right)=\tau_{c_{q}}^{-1} \tau_{q, p} \tau_{c_{p}} v_{0}$ and

$$
\left\langle H_{c}\left(v_{0}\right), e_{i}^{p_{0}}\right\rangle=\left\langle\sum_{j=1}^{n} \tau_{q, p} e_{j}^{p}, e_{i}^{q}\right\rangle=\sum_{j=1}^{n} A(p, q)_{i j} v_{j} .
$$

Combining this last equality with (4.2) we obtain $\alpha^{\prime}\left|v_{0}\right| \geq\left|D_{A} f(p, q)\right|$. Finally, computing $\left\|D_{A} f\right\|^{2}$ leads to

$$
\left\|D_{A} f\right\|^{2} \leq \frac{1}{2} \alpha^{\prime 2} \nu\|f\|^{2} .
$$

So that the second part of the theorem follows.

Appendix A. Technical tools. The following lemma is a generalization of Lemma 11.1 in [20] and a local version of Lemma 0.1 of [27]. 
Lemma A.1. Let $M \in \mathcal{M}\left(m, \kappa, r_{0}\right)$ and $u$ a non-negative function on the ball $B(p, R)$, with $R<\frac{1}{2} r_{0}$, such that $\Delta u \leq \alpha u+\beta$. Let $0<\theta<1$. Then there exist $c_{1}$, $c_{2}, c_{3}>0$ (depending only on $m, n, \kappa, R, \alpha$ and $\beta$ ) and $0<c(m)<s \leq 1$ such that

$$
\|u\|_{\infty, \theta R} \leq\left(\left(c_{1}+c_{2} \frac{1}{(1-\theta)^{2}}\right)^{c_{3}}\|u\|_{2, R}\right)^{s}
$$

where $\|u\|_{\infty, \theta R}=\sup \{u(x) \mid x \in B(p, \theta R)\}$, and $\|u\|_{q, R}^{q}=\int_{B(p, R)} u^{q}(x) d V(x)$.

Note that, if $\beta=0$ then $s=1$ (see [20], Lemma 11.1).

Proof. The proof combines the proof given in [20] (Lemma 11.1) and Lemma 0.1 of [27]. Let $u: B(p, R) \rightarrow \mathbb{R}, u \geq 0$ such that $\Delta u \leq \alpha u+\beta$. Let $\nu=\frac{m}{2}$ if $m \geq 3$ and $\nu=2$ otherwise. Let $\mu$ be such that $\frac{1}{\mu}+\frac{1}{\nu}=1$. For $0<\rho<\rho+\sigma<R$, let $\phi_{\rho, \sigma}$ be the Lipschitz cut-off function depending only on the distance to $p$ given by

$$
\phi_{\rho, \sigma}(r)=\phi(r)= \begin{cases}0 & \text { on } B(p, R) \backslash B(p, \rho+\sigma), \\ \frac{\rho+\sigma+r}{\sigma} & \text { on } B(p, \rho+\sigma) \backslash B(p, \rho), \\ 1 & \text { on } B(p, \rho) .\end{cases}
$$

Then for an arbitrary constant $a \geq 1$, we have

$$
\left\|u^{2 a}\right\|_{\mu, \rho} \leq\left\|\phi u^{a}\right\|_{2 \mu}^{2} .
$$

As the injectivity radius of $M$ is bounded below $\left(\operatorname{Inj}(M) \geq r_{0}>0\right)$ and the Ricci curvature too $(\operatorname{Ricci}(M, g) \geq-(m-1) \kappa g)$ Sobolev embeddings for complete manifolds are valid and we can apply the Sobolev inequalities to $\left\|\phi u^{a}\right\|_{2 \mu}^{2}$ (see [16], Theorem 3.3). More precisely, there exists a constant $c_{s}>0$ depending only on $m, \kappa$ and $r_{0}$ such that

$$
\left\|\phi u^{a}\right\|_{2 \mu}^{2} \leq c_{s}\left(\left\|d\left(\phi u^{a}\right)\right\|_{2}^{2}+\left\|\phi u^{a}\right\|_{2}^{2}\right) .
$$

Replacing $c_{s}$ by $C R^{2}$, we can rewrite the inequality as

$$
\left\|\phi u^{a}\right\|_{2 \mu}^{2} \leq C R^{2}\left(\left\|d\left(\phi u^{a}\right)\right\|_{2}^{2}+\left\|\phi u^{a}\right\|_{2}^{2}\right) .
$$

Therefore,

$$
\left\|u^{2 a}\right\|_{\mu, \rho} \leq C R^{2}\left(\left\|d\left(\phi u^{a}\right)\right\|_{2}^{2}+\left\|\phi u^{a}\right\|_{2}^{2}\right) .
$$

However

$$
\int_{M}\left|d\left(\phi u^{a}\right)\right|^{2} d V \leq a \int_{M} \phi^{2} u^{2 a-1} \Delta u d V+\int_{M}|d \phi|^{2} u^{2 a} d V
$$

(see [20], p.81). Hence using the assumption on $\Delta u$ and $u \geq 0$ we obtain

$$
\begin{aligned}
\left\|u^{2 a}\right\|_{\mu, \rho} \leq C R^{2}\left(\int_{M} \phi u^{2 a} d V+a \alpha \int_{M} \phi^{2} u^{2 a} d V\right. & \\
& \left.\quad+a \beta \int_{M} \phi^{2} u^{2 a-1} d V+\int_{M}|d \phi|^{2} u^{2 a} d V\right)
\end{aligned}
$$


and by construction of $\phi$, we obtain

$$
\begin{aligned}
\left\|u^{2 a}\right\|_{\mu, \rho} \leq C & R^{2}\left(a \alpha+\frac{1}{\sigma^{2}}+1\right) \int_{B(p, \rho+\sigma)} u^{2 a} d V+C R^{2} a \beta \int_{B(p, \rho+\sigma)} u^{2 a-1} d V \\
\leq & C R^{2}\left(a \alpha+\frac{1}{\sigma^{2}}+1\right)\|u\|_{2 a, \rho+\sigma}^{2 a}+C R^{2} a \beta V(p, \rho+\sigma)^{\frac{1}{2 a}}\|u\|_{2 a, \rho+\sigma}^{2 a-1} .
\end{aligned}
$$

Finally, we have shown that for any $a \geq 1,0<\rho<\rho+\sigma<R$, we have

$$
\|u\|_{2 a \mu, \rho}^{2 a} \leq C R^{2}\left(a \alpha+\frac{1}{\sigma^{2}}+1\right)\|u\|_{2 a, \rho+\sigma}^{2 a}+C R^{2} a \beta V(p, \rho+\sigma)^{\frac{1}{2 a}}\|u\|_{2 a, \rho+\sigma}^{2 a-1} .
$$

This was the first step of the proof. Now, we will proceed with a Moser iteration. To that aim, let

$$
\begin{gathered}
a_{0}=1, a_{1}=\frac{m}{m-2}=\mu, \ldots, a_{i}=\mu^{i}, \ldots \\
\sigma_{0}=\frac{1-\theta}{2} R, \sigma_{1}=\frac{1-\theta}{4} R, \ldots, \sigma_{i}=\frac{1-\theta}{2^{i+1}} R, \ldots \\
\rho_{0}=R-\sigma_{0}, \rho_{1}=R-\sigma_{0}-\sigma_{1}, \ldots, \rho_{i}=R-\sum_{j=0}^{i} \sigma_{j}, \ldots
\end{gathered}
$$

and $\rho_{-1}=R$. Observe that $\rho_{i}>\theta R$ for any $i$ and $\rho_{i} \rightarrow \theta R$ as $i \rightarrow \infty$. Moreover, for any $A_{i}, B_{i}>0$

$$
\begin{aligned}
& \left(A_{i}+B_{i}\right) \min \left\{\|u\|_{2 a_{i}, \rho_{i}+\sigma_{i}}^{2 a_{i}},\|u\|_{2 a_{i}, \rho_{i}+\sigma_{i}}^{2 a_{i}}\right\} \leq \\
& A_{i}\|u\|_{2 a_{i}, \rho_{i}+\sigma_{i}}^{20 a_{i}}+B_{i}\|u\|_{2 a_{i}, \rho_{i}+\sigma_{i}}^{2 a_{a_{i}-1}} \leq\left(A_{i}+B_{i}\right)\|u\|_{2 a_{i}, \rho_{i}+\sigma_{i}}^{b_{i}}
\end{aligned}
$$

where $b_{i}$ is suitably chosen $\left(b_{i} \in\left\{2 a_{i-1}, 2 a_{i}\right\}\right)$. Now replace above $a, \rho, \sigma$ by $a_{i}$ respectively $\rho_{i}, \sigma_{i}$ to obtain

$$
\|u\|_{2 a_{i+1}, \rho_{i}} \leq\left(C R^{2}\left(a_{i} \alpha+\frac{1}{\sigma_{i}^{2}}+1+a_{i} \beta V\left(p, \rho_{i-1}\right)^{\frac{1}{2 a_{i}}}\right)\right)^{\frac{1}{2 a_{i}}}\|u\|_{2 a_{i}, \rho_{i-1}}^{\frac{b_{i}}{2 a_{i}}} .
$$

Then iterate this inequality to obtain (using Bishop-Gromov comparison theorem, Croke's inequality and $a_{i} \geq 1$ )

$$
\|u\|_{\infty, \theta R} \leq c\left(\prod_{i=0}^{\infty}\left(C R^{2}\left(a_{i} \alpha+a_{i} c^{\prime} \beta+1\right)+C \frac{R^{2}}{\sigma_{i}^{2}}\right)^{\frac{1}{2 a_{i}}}\|u\|_{2, R}^{\frac{b_{0}}{2}}\right)^{\prod_{j=1}^{\infty} \frac{b_{j}}{2 a_{j}}} .
$$

By the same argument as in [27], $\prod_{j=0}^{\infty} \frac{b_{j}}{2 a_{j}}$ converges to $s \in\left[e^{-(n-2) \frac{\ln (2)}{2}}, 1\right]$. It remains then to show that $\prod_{i=0}^{\infty}\left(C R^{2}\left(a_{i} \alpha+a_{i} c^{\prime} \beta+1\right)+C \frac{R^{2}}{\sigma_{i}^{2}}\right)^{\frac{1}{2 a_{i}}}$ converges too. But we have that $\prod_{i=0}^{\infty} B^{\mu^{-i}}=B^{\frac{\mu}{\mu-1}}($ as $\mu>1)$ and $\sum_{i=0}^{\infty} i \mu^{-i}$ is finite, therefore

$$
\begin{gathered}
\prod_{i=0}^{\infty}\left(C R^{2} \mu^{i}\left(\alpha+c^{\prime} \beta+\mu^{-i}\right)+4 C \frac{4^{i}}{(1-\theta)^{2}}\right)^{\frac{1}{2 \mu^{2}}} \leq \\
\prod_{i=0}^{\infty} \max \{\mu, 4\}^{\frac{i}{2 \mu^{2}}}\left(C R^{2}\left(\alpha+c^{\prime} \beta+1\right)+C \frac{4}{(1-\theta)^{2}}\right)^{\frac{1}{2 \mu^{2}}} \\
\leq c(\mu)\left(C R^{2}\left(\alpha+c^{\prime} \beta+1\right)+C \frac{4}{(1-\theta)^{2}}\right)^{\frac{1}{2} \frac{\mu}{\mu-1}} .
\end{gathered}
$$


This implies the claim.

A.1. Proof of Lemma 3.19. The proof differs according to the assumptions made on $E$.

Assume E is of harmonic curvature. By Remark 3.13 and Remark 3.14, we have

$$
\begin{aligned}
& \sum_{i=1}^{n} f_{i}(p) e_{i}^{p}(x)-\sum_{i=1}^{n} f_{i}(q) e_{i}^{q}(x)= \\
& \sum_{i=1}^{n} f_{i}(p)\left(e_{i}^{p}(x)-\tau_{x, p} e_{i}^{p}(p)\right)+D_{A} f(q, p)_{i} \tau_{x, p} e_{i}^{p}(p)+f_{i}(q)\left(\tau_{x, p} e_{i}^{q}(p)-e_{i}^{q}(x)\right) .
\end{aligned}
$$

By Lemma 3.3 and as $d^{*} R^{E}=0,\left|e_{i}^{p}(x)-\tau_{x, p} e_{i}^{p}\right|^{2} \leq c \lambda_{i}(p)$ for $1 \leq i \leq \mu(p)$ and $\left|\tau_{x, p} e_{i}^{q}(p)-e_{i}^{q}(x)\right|^{2} \leq c \lambda_{i}(q)$ for $1 \leq i \leq \mu(q)$. Moreover if $\mu(q)<i \leq n, \mid \tau_{x, p} e_{i}^{q}(p)-$ $\left.e_{i}^{q}(x)\right|^{2} \leq 4$. Therefore

$$
\begin{aligned}
& \mid \sum_{i=1}^{n} f_{i}(p) e_{i}^{p}(x)-\sum_{i=1}^{n} f_{i}(q) e_{i}^{q}(x)\left.\right|^{2} \leq \\
& c^{\prime}\left(\left|D_{A} f(q, p)\right|^{2}+(V f)(p) \cdot f(p)+(V f)(q) \cdot f(q)\right)
\end{aligned}
$$

which implies the lemma in this case.

Assume $\mathbf{E}$ is of rank one, then

$$
\begin{aligned}
\sum_{i=1}^{n} f_{i}(p) e_{i}^{p}(x) & -\sum_{i=1}^{n} f_{i}(q) e_{i}^{q}(x)= \\
& \sum_{i=1}^{n} D_{A} f(q, p)_{i} e_{i}^{p}(x)+\sum_{j=1}^{n} f_{j}(q) \sum_{i=1}^{n} e_{i}^{p}(x)\left(A(q, p)_{i j}-a(q, p)_{i j}(x)\right) .
\end{aligned}
$$

By definition of $A(q, p)_{i j}$ and by the work of Buser (Lemma 5.1 in [8]) there exists $c_{B}>0$ depending only on $m, \kappa$ and $\varepsilon$ such that

$$
\int_{B_{p q}}\left|A(q, p)_{i j}-a(q, p)_{i j}(x)\right|^{2} d V(x) \leq c_{B} \int_{B_{p q}}\left|d a(q, p)_{i j}(x)\right|^{2} d V(x) .
$$

Moreover

$$
\begin{aligned}
\left(1-\delta^{\prime}\right) \sum_{i=1}^{n}\left|d a(q, p)_{i j}(x)\right|^{2} \leq & \\
\left|\sum_{i=1}^{n} d a(q, p)_{i j}(x) e_{i}^{p}(x)\right|^{2}=\mid \nabla e_{j}^{q}(x) & -\left.\sum_{i=1}^{n} a(q, p)_{i j}(x) \nabla e_{i}^{p}(x)\right|^{2} \\
& \leq c\left(\left|\nabla e_{j}^{q}(x)\right|^{2}+\sum_{i=1}^{n}\left|\nabla e_{i}^{p}(x)\right|^{2}\right) .
\end{aligned}
$$


As the bundle is of rank one, $\lambda_{1}(p)=\ldots=\lambda_{n}(p)$. Therefore $\lambda_{1}(p) \leq \delta$ implies $\int_{B(p, 10 \varepsilon)}\left|\nabla e_{i}^{p}(x)\right|^{2} d V(x) \leq c \lambda_{1}(p)$. Otherwise $\int_{B(p, 10 \varepsilon)}\left|\nabla e_{i}^{p}(x)\right|^{2} d V(x) \leq c \leq$ $c \delta^{-1} \lambda_{1}(p)$ by Lemma 3.12, which implies

$$
\int_{B_{p q}}\left|A(q, p)_{i j}-a(q, p)_{i j}(x)\right|^{2} d V(x) \leq c^{\prime}\left(\lambda_{1}(p)+\lambda_{1}(q)\right) .
$$

Hence

$$
\begin{aligned}
& \int_{B(q, \varepsilon)}\left|\sum_{i=1}^{n} f_{i}(p) e_{i}^{p}(x)-\sum_{i=1}^{n} f_{i}(q) e_{i}^{q}(x)\right|^{2} \leq \\
& c^{\prime \prime}\left(\left|D_{A} f(q, p)\right|^{2}+|f(q)|^{2}\left(\lambda_{1}(p)+\lambda_{1}(q)\right)\right) .
\end{aligned}
$$

This concludes the proof of Lemma 3.19.

A.2. Proof of Lemma 3.22. The proof differs according to the assumptions made on $E$.

Assume $\mathbf{E}$ is of harmonic curvature. As $\left\{\tau_{y, p} e_{i}^{p}(p)\right\}_{i=1}^{n}$ is an almost orthonormal basis and by Remark 3.14

$$
\begin{aligned}
& \sum_{i=1}^{n}\left|s_{i}^{p}(y)-\sum_{j=1}^{n} A(q, p)_{i j} s_{j}^{q}(y)\right|^{2} \leq \\
& \quad\left(1-\delta^{\prime}\right)^{-1}\left|\sum_{i=1}^{n} s_{i}^{p}(y)\left(\tau_{y, p} e_{i}^{p}(p)-e_{i}^{p}(y)\right)+\sum_{i=1}^{n} s_{i}^{q}(y)\left(e_{i}^{q}(y)-\tau_{y, p} e_{i}^{q}(p)\right)\right|^{2} .
\end{aligned}
$$

Integrate then over $B_{p q}^{\prime}$ and apply Lemma 3.3 to obtain

$$
\sum_{i=1}^{n} \int_{B_{p q}^{\prime}}\left|s_{i}^{p}(y)-\sum_{j=1}^{n} A(q, p)_{i j} s_{j}^{q}(y)\right|^{2} d V(y) \leq c((\widetilde{V} s)(p)+(\widetilde{V} s)(q)) .
$$

Assume E is of rank one. Recall that $s_{i}^{p}(y)=\sum_{j=1}^{n} a(q, p)_{i j}(y) s_{j}^{q}(y)$. Hence

$$
s_{i}^{p}(y)-\sum_{j=1}^{n} A(q, p)_{i j} s_{j}^{q}(y)=\sum_{j=1}^{n}\left(a(q, p)_{i j}(y)-A(q, p)_{i j}\right) s_{j}^{q}(y) .
$$

Therefore

$$
\begin{aligned}
\int_{B_{p q}^{\prime}}\left|s_{i}^{p}(y)-\sum_{j=1}^{n} A(q, p)_{i j} s_{j}^{q}(y)\right| d V(y) \leq \\
\|s\|_{2,3 \varepsilon} \sum_{j=1}^{n}\left(\int_{B_{p q}^{\prime}}\left|a(q, p)_{i j}(y)-A(q, p)_{i j}\right|^{2}\right)^{\frac{1}{2}} d V(y) .
\end{aligned}
$$


Finally, as $B_{p q}^{\prime} \subset B_{p q}$, inequality (A.1) implies

$$
\sum_{i=1}^{n}\left(\int_{B_{p q}^{\prime}}\left|s_{i}^{p}(y)-\sum_{j=1}^{n} A(q, p)_{i j} s_{j}^{q}(y)\right| d V(y)\right)^{2} \leq c(\widetilde{V} s)(p)
$$

and this concludes the proof of Lemma 3.22.

\section{REFERENCES}

[1] Aubry E., Colbois B., Ghanaat P., Ruh E. A., Curvature, Harnack's Inequality, and a Spectral Characterization of Nilmanifolds, Ann. Global Anal. Geom., 23 (2003), pp. 227246.

[2] Ballmann W., Brüning J., Carron G., Eigenvalues and holonomy, Int. Math. Res. Not., 12 (2003), pp. 657-665.

[3] BÉRARD P. H., From vanishing theorems to estimating theorems: The Bochner method revisited, Bull. Amer. Math. Soc., 19 (1988), pp. 371-406.

[4] Bollobás B., Modern Graph Theory, Grad. Texts in Math., Springer-Verlag, 1998.

[5] Brooks R., The spectral geometry of a tower of coverings, J. Differential Geometry, 23 (1986), pp. $97-107$.

[6] Burger M., Estimation de petites valeurs propres du laplacien d'un revêtement de variétés riemanniennes compactes, C. R. Acad. Sci Paris Sr. I Math., 302 (1986), pp. 191-194.

[7] Buser P., On Cheeger's inequality, Proc. of Sympos. in Pure Math., Amer. Math. Soc., 36 (1980).

[8] Buser P., A note on the isoperimetric constant, Ann. Scient. ENS, 15 (1982), pp. 213-230 .

[9] Buser P., On the bipartition of graphs, Discrete Appl. Math., 9 (1984), pp. 105-109.

[10] Chavel I., Isoperimetric Inequalities. Differential Geometric and Analytic Perspectives, Cambridge Tracts in Math., Cambridge Univ. Press, 2001.

[11] Dodziuk J., Eigenvalues of the Laplacian on Forms, Proceedings of the AMS, 85 (1982).

[12] Donaldson S. K., Kronheimer P. B., The Geometry of Four-Manifolds, Oxford Math. Monogr., Oxford Science Publications, 1990.

[13] Freed D. S., Uhlenbeck K. K., Instantons and Four-Manifolds, Math. Sci. Res. Inst. Publ., Springer-Verlag, 1984.

[14] Gilbarg D., Trudinger N. S., Elliptic Partial Differential Equations of Second Order, Classics in Mathematics, Springer, 2001.

[15] Gilkey P. B., Invariance Theory, the Heat Equation, and the Atiyah-Singer Index Theorem, Stud. Adv. Math., CRC Press, 1995.

[16] Hebey E., Nonlinear Analysis on Manifolds : Sobolev Spaces and Inequalities, Courant Lect. Notes Math. 5, CIMS, 1999.

[17] Hebey E., Sobolev Spaces on Riemannian Manifolds, Lecture Notes in Mathematics, Springer, 1996.

[18] Kanai M., Rough isometries, and combinatorial approximations of geometries of non-compact Riemannian manifolds, J. Math. Soc. Japan, 37 (1985).

[19] Kanai M., Analytic inequalities, and rough isometries between non-compact Riemannian manifolds, urvature and Topology of Riemannian Manifolds (Katata 1985), 122-137, Lecture Notes in Math., 1201, Springer, 1986.

[20] Li P., Lecture Notes on Geometric Analysis, Lecture Notes Series No. 6, Research Institute of Mathematics, Global Analysis Research Center, Seoul National University, 1993.

[21] Lubotsky A., Discrete Groups, Expanding Graphs and Invariant Measures, Birkhäuser, 1994.

[22] Mantuano T., Discretization of compact Riemannian manifolds applied to the spectrum of Laplacian, Ann. Global Anal. Geom., 27 (2005), pp. 33-46.

[23] Mathai V., Yates S., Approximating Spectral Invariants of Harper Operators on Graphs, J. Funct. Anal., 188 (2002), pp. 111-136.

[24] Morita S., Geometry of Differential Forms, Translations of Mathematical Monographs, American Mathematical Society, 2001.

[25] Petersen P., Riemannian Geometry, Grad. Texts in Math., Springer-Verlag, 1998.

[26] Petersen P., Sprouse C., Eigenvalue pinching for Riemannian vector bundles, J. Reine Angew. Math., 511 (1999), pp. 73-86.

[27] Petersen P., Sprouse C., Erratum to Eigenvalue pinching for Riemannian vector bundles, Preprint.

[28] Sakai T., Riemannian Geometry, American Math. Soc., 1997. 
T. MANTUANO 